\title{
Type B 3-fold Supersymmetry and Non-polynomial Invariant Subspaces
}

\author{
Toshiaki Tanaka* \\ Institute of Particle and Nuclear Studies, \\ High Energy Accelerator Research Organization (KEK), \\ 1-1 Oho, Tsukuba, Ibaraki 305-0801, Japan ${ }^{\dagger}$
}

\begin{abstract}
We obtain the most general type B 3-fold supersymmetry by solving directly the intertwining relation. We then show that it is a necessary and sufficient condition for a second-order linear differential operator to have three linearly independent local analytic solutions. We find that there are eight linearly independent non-trivial linear differential operators of this kind. As a by-product, we find new quasi-solvable second-order operators preserving a monomial or polynomial subspace, one in type $\mathrm{B}$, two in type $\mathrm{C}$, and four in type $X_{2}$, all of which have been missed in the existing literature. In addition, we show that type $\mathrm{A}$, type $\mathrm{B}$, and type $\mathrm{C}$ 3-fold supersymmetries are connected continuously via one parameter. A few new quasi-solvable models are also presented.
\end{abstract}

PACS numbers: 02.30.Hq; 03.65.Ca; 03.65.Ge; 11.30.Pb

Keywords: $\mathcal{N}$-fold supersymmetry; Quasi-solvability; Schrödinger operators; Invariant spaces; Monomial subspaces; Polynomial subspaces

*Electronic address: tanaka.toshiaki@ocha.ac.jp

${ }^{\dagger}$ Department of Physics, Faculty of Science, Ochanomizu University, 2-1-1 Ohtsuka, Bunkyo-ku, Tokyo 112-8610, Japan 


\section{INTRODUCTION}

Since the discovery of the equivalence between $\mathcal{N}$-fold supersymmetry (SUSY) and weak quasi-solvability [1] in 2001, the research activity on exactly solvable Schrödinger operators has come into a new era. Indeed, we now recognize that all the exactly solvable ones whose solutions are expressible in terms of a classical orthogonal polynomial system are characterized as a particular case of type A $\mathcal{N}$-fold SUSY $[2,3]$ and that the ones which admit two linearly independent series solutions belong to type $\mathrm{C} \mathcal{N}$-fold SUSY [4]. In addition, a particular class of type B $\mathcal{N}$-fold SUSY constructed in Ref. [5] is now placed in a set of second-order linear differential operators preserving a so-called exceptional polynomial subspace of codimension one [6,7]. A set of new quasi-solvable operators preserving an exceptional polynomial subspace of codimension two was also constructed in the framework of $\mathcal{N}$-fold SUSY [8], which is called type $X_{2}$. For the development of $\mathcal{N}$-fold SUSY until the middle of 2000s and the terminology, see the review [9]. For its non-perturbative aspects, see also the review [10].

The mathematical structure of $\mathcal{N}$-fold SUSY is characterized by a higher-order intertwining relation if it is represented by differential operators. In general, however, it is quite difficult to solve directly the relation under consideration which actually consists of simultaneous coupled nonlinear differential equations. Until now on, the most general form of $\mathcal{N}$-fold SUSY is known only up to $\mathcal{N}=4$ [11]. They are all weakly quasi-solvable, that is, they keep a linear subspace annihilated by the corresponding $\mathcal{N}$-fold supercharge invariant. It does not immediately mean, however, that some local solutions are available in a closed form. In other words, it does not automatically guarantee quasi-solvability in the strong sense.

Hence, a natural question would arise; under what conditions an $\mathcal{N}$-fold SUSY system admits a number of local analytic solutions. In the case where the number is two, it was proved [12] that a necessary and sufficient condition for it is to have type A 2-fold SUSY. The latter fact further enabled one to construct systematically shape-invariant potentials within this type of symmetry $[13,14]$. These achievements clearly show the power of $\mathcal{N}$-fold SUSY, and we expect that we would be able to step forward to more general cases, beginning with the case where the number of available analytic solutions is three.

On the other hand, among the aforementioned four types of $\mathcal{N}$-fold SUSY which are different with each other for $\mathcal{N}>2$, type $\mathrm{A}$ is the only one for which a necessary and sufficient condition is known. It seems that it is still quite hard to find it for an arbitrary $\mathcal{N}>2$ even if we restrict our consideration to a particular type. Hence, it would be a realistic plan to make the examination from lower to higher values of $\mathcal{N}$ step by step until when we eventually discover, if it exists, an inductive way toward a general statement applicable to an arbitrary $\mathcal{N}>2$ case.

In this paper, we examine under what conditions a one-body quantum Hamiltonian would admit three linearly independent local solutions in closed form. In addition, we investigate what type of 3-fold SUSY such a system must possess as a consequence of the equivalence between $\mathcal{N}$-fold SUSY and weak quasi-solvability. We show that it is the most general type B, for which only a particular case has been so far explored. In other words, we prove that type B 3-fold SUSY is a necessary and sufficient condition for a Hamiltonian to admit three linearly independent local analytic solutions. We find that there are eight linearly independent second-order linear differential operators of this kind. As a by-product of this finding, we obtain some new quasi-solvable operators preserving a three-dimensional 
monomial or polynomial subspace which have not been considered in the literature. More precisely, we discover one new quasi-solvable operator for a type B monomial space, two for type $\mathrm{C}$, and four for type $X_{2}$. Furthermore, we find that all the eight quasi-solvable operators in the type $\mathrm{A}$, type $\mathrm{B}$, and type $\mathrm{C}$ cases are connected continuously via a one parameter.

We organize the paper as follows. In Section II, we first construct the most general second-order linear differential operator which preserves a three-dimensional linear function space. In Section III, we solve directly the intertwining relation in type B 3-fold SUSY to obtain a necessary and sufficient condition for it. We then show that it leads to the operator which is identical with the one obtained in Section II. Sections IV and V deal with particular cases of the most general type B SUSY. In Section IV, we present three examples of type B 3-fold SUSY models obtained by a particular choice of the three-dimensional linear function space. In Section V, we consider the cases where the three-dimensional linear space is given by type $\mathrm{A}$, type $\mathrm{B}$, and type $\mathrm{C}$ monomial subspaces. We show there that these three different types are continuously connected via a parameter. We also find new quasi-solvable operators preserving type $\mathrm{B}$ and type $\mathrm{C}$ monomial subspaces. In Section VI, we examine commutation relations of quasi-solvable operators. The analysis demonstrates a reason why the family of type A models is special in the sense that it is Lie-algebraic. In Section VII, we first reformulate the most general type B 3-fold SUSY system in Section III, and then apply it to the case of three-dimensional $X_{2}$ polynomial subspaces. In Section VIII, we summarize the results and discuss their implications and prospects for the future studies.

\section{QUASI-SOLVABLE OPERATORS PRESERVING A THREE-DIMENSIONAL SPACE}

Let us first consider a one-dimensional quantum Hamiltonian $H$ which admits three linearly independent local analytic solutions in closed form to the corresponding Schrödinger equation,

$$
H \psi_{i}(q)=E_{i} \psi_{i}(q) \quad(i=1,2,3),
$$

where each of $\psi_{i}$ does not necessarily normalizable. It is evident that the Hamiltonian $H$ preserves a three-dimensional linear space $\mathcal{V}_{3}$ of functions defined by

$$
\mathcal{V}_{3}=\left\langle\psi_{1}(q), \psi_{2}(q), \psi_{3}(q)\right\rangle \text {. }
$$

Conversely, if a Hamiltonian preserves a linear space (2.2) spanned by three known functions, we can diagonalize it in the three-dimensional space algebraically, and thus obtain three linearly independent local solutions to the corresponding Schrödinger equation. An available set of three functions differs depending on the Hamiltonian under consideration, but we can extract a common mathematical structure shared by all such systems. For this purpose, let us introduce a "gauged" Hamiltonian $\tilde{H}^{-}$by $\tilde{H}^{-}=\psi_{1}(q) H \psi_{1}(q)^{-1}$. Then, it immediately follows from the invariance $H \mathcal{V}_{3} \subset \mathcal{V}_{3}$ that the gauged Hamiltonian $\tilde{H}^{-}$preserves the linear space

$$
\tilde{\mathcal{V}}_{3}^{-}[z]=\langle 1, z, f(z)\rangle,
$$

where the variable $z$ and the function $f(z)$ are defined by

$$
z=\psi_{2}(q) / \psi_{1}(q), \quad f(z)=\psi_{3}(q) / \psi_{1}(q) .
$$


It is evident from the assumed linear independence of $\psi_{i}(q)(i=1,2,3)$ that $f(z)$ is arbitrary with the exception of a polynomial of at most first degree in $z$. That is, $f(z)$ is an arbitrary function satisfying $f^{\prime \prime}(z) \neq 0$. In addition, it is not necessary for the function $f(z)$ to admit an explicit form in terms of the variable $z$. As we shall see in Section III, the specific choice of the variable $z$, which results in the form of the vector space (2.3), enables us to establish easily a connection with an existing type of 3-fold SUSY. Later in Section VII, we shall reconsider the problem with the most general change of variable.

From the above argument, it is now clear that any one-dimensional quantum Hamiltonian $H$ in the physical $q$-space which has three linearly independent local analytic solutions can be converted with a gauge transformation and a change of variable $z=z(q)$ to a gauged Hamiltonian $\tilde{H}^{-}$in the gauged $z$-space which leaves the linear space (2.3) invariant. Such a gauged Hamiltonian $\tilde{H}^{-}$is no longer a Schrödinger operator but is a general linear secondorder differential operator in the variable $z$ :

$$
\tilde{H}^{-}=-A(z) \frac{\mathrm{d}^{2}}{\mathrm{~d} z^{2}}-B(z) \frac{\mathrm{d}}{\mathrm{d} z}-C(z)
$$

where $A(z), B(z)$, and $C(z)$ are functions of $z$ to be determined. To preserve the space $(2.3)$, it is necessary and sufficient for $\tilde{H}^{-}$to satisfy

$$
\begin{aligned}
\tilde{H}^{-} 1 & =-C(z)=-c_{2} f(z)-c_{1} z-c_{0}, \\
\tilde{H}^{-} z & =-B(z)-C(z) z=-b_{2} f(z)-b_{1} z-b_{0}, \\
\tilde{H}^{-} f(z) & =-A(z) f^{\prime \prime}(z)-B(z) f^{\prime}(z)-C(z) f(z)=-a_{2} f(z)-a_{1} z-a_{0},
\end{aligned}
$$

where $c_{i}, b_{i}$, and $a_{i}(i=0,1,2)$ are all constants. From these conditions, we obtain

$$
\begin{aligned}
A(z) f^{\prime \prime}(z)= & {\left[\left(c_{2} z-b_{2}\right) f(z)+c_{1} z^{2}+\left(c_{0}-b_{1}\right) z-b_{0}\right] f^{\prime}(z) } \\
& -\left[c_{2} f(z)+c_{1} z+c_{0}-a_{2}\right] f(z)+a_{1} z+a_{0}, \\
B(z)= & -\left(c_{2} z-b_{2}\right) f(z)-c_{1} z^{2}-\left(c_{0}-b_{1}\right) z+b_{0}, \\
C(z)= & c_{2} f(z)+c_{1} z+c_{0} .
\end{aligned}
$$

Substituting the latter formulas for $A(z), B(z)$, and $C(z)$ into $(2.5)$, we see that $\tilde{H}^{-}$is expressed as

$$
\begin{aligned}
\tilde{H}^{-}= & -c_{2} J_{8}-c_{1} J_{7}+b_{2} J_{6}+\left(b_{1}-c_{0}\right) J_{5}+b_{0} J_{4} \\
& -\left(a_{2}-c_{0}\right) J_{3}-a_{1} J_{2}-a_{0} J_{1}-c_{0},
\end{aligned}
$$

where $J_{i}(i=1, \ldots, 8)$ are given by

$$
\begin{aligned}
& J_{1}[z]=\frac{1}{f^{\prime \prime}(z)} \frac{\mathrm{d}^{2}}{\mathrm{~d} z^{2}}, \quad J_{2}[z]=z J_{1}[z]=\frac{z}{f^{\prime \prime}(z)} \frac{\mathrm{d}^{2}}{\mathrm{~d} z^{2}}, \quad J_{3}[z]=f(z) J_{1}[z]=\frac{f(z)}{f^{\prime \prime}(z)} \frac{\mathrm{d}^{2}}{\mathrm{~d} z^{2}}, \\
& J_{4}[z]=\frac{f^{\prime}(z)}{f^{\prime \prime}(z)} \frac{\mathrm{d}^{2}}{\mathrm{~d} z^{2}}-\frac{\mathrm{d}}{\mathrm{d} z}, \quad J_{5}[z]=z J_{4}[z]=\frac{z f^{\prime}(z)}{f^{\prime \prime}(z)} \frac{\mathrm{d}^{2}}{\mathrm{~d} z^{2}}-z \frac{\mathrm{d}}{\mathrm{d} z}, \\
& J_{6}[z]=f(z) J_{4}[z]=\frac{f(z) f^{\prime}(z)}{f^{\prime \prime}(z)} \frac{\mathrm{d}^{2}}{\mathrm{~d} z^{2}}-f(z) \frac{\mathrm{d}}{\mathrm{d} z}, \\
& J_{7}[z]=z J_{9}[z]=\frac{z f^{\prime}(z)-f(z)}{f^{\prime \prime}(z)} z \frac{\mathrm{d}^{2}}{\mathrm{~d} z^{2}}-z^{2} \frac{\mathrm{d}}{\mathrm{d} z}+z, \\
& J_{8}[z]=f(z) J_{9}[z]=\frac{z f^{\prime}(z)-f(z)}{f^{\prime \prime}(z)} f(z) \frac{\mathrm{d}^{2}}{\mathrm{~d} z^{2}}-z f(z) \frac{\mathrm{d}}{\mathrm{d} z}+f(z),
\end{aligned}
$$


and $J_{9}$ is defined by

$$
J_{9}[z]=J_{5}[z]-J_{3}[z]+1=\frac{z f^{\prime}(z)-f(z)}{f^{\prime \prime}(z)} \frac{\mathrm{d}^{2}}{\mathrm{~d} z^{2}}-z \frac{\mathrm{d}}{\mathrm{d} z}+1 .
$$

Therefore, a linear space of non-trivial differential operators of at most second order which preserves the space (2.3) is spanned by the eight linearly independent operators $J_{i}(i=$ $1, \ldots, 8)$. This result is indeed consistent with the general ones studied in Ref. [15].

It is worth noting that there are in general no first-order linear differential operators which preserves (2.3). In fact, we can easily see that Eq. (2.9) provides a constraint on the function $f(z)$ when we set $A(z)=0$ to consider a first-order quasi-solvable operator. This constraint cannot be satisfied unless $f(z)$ has certain specific forms, cf. Sections V and VI.

\section{TYPE B 3-FOLD SUSY}

Type B $\mathcal{N}$-fold SUSY was first discovered in Ref. [5] by a simple deformation of type A $\mathcal{N}$-fold supercharge. The component of type B 3 -fold supercharge is given by

$$
P_{3}^{-}=\left(\frac{\mathrm{d}}{\mathrm{d} q}+W(q)-E(q)-F(q)\right)\left(\frac{\mathrm{d}}{\mathrm{d} q}+W(q)\right)\left(\frac{\mathrm{d}}{\mathrm{d} q}+W(q)+E(q)\right)
$$

where the three functions $W(q), E(q)$, and $F(q)$ are at present arbitrary. A pair of Hamiltonians,

$$
H^{ \pm}=-\frac{1}{2} \frac{\mathrm{d}^{2}}{\mathrm{~d} q^{2}}+V^{ \pm}(q)
$$

is said to have type B 3-fold SUSY if it is intertwined by $P_{3}^{-}$in (3.1) as

$$
P_{3}^{\mp} H^{\mp}=H^{ \pm} P_{3}^{\mp},
$$

where $P_{3}^{+}$is the transposition of $P_{3}^{-}$in the $q$-space, $P_{3}^{+}=\left(P_{3}^{-}\right)^{\mathrm{T}}$. A direct calculation shows that the intertwining relation (3.3) holds if and only if the potential terms in (3.2) have the following form

$$
V^{ \pm}=\frac{1}{2} W^{2}-\frac{1}{3}\left(2 E^{\prime}-E^{2}\right)-\frac{1}{6}\left(2 F^{\prime}+2 W F-2 E F-F^{2}\right) \pm \frac{1}{2}\left(3 W^{\prime}-F^{\prime}\right),
$$

and simultaneously the three functions $W(q), E(q)$, and $F(q)$ satisfy

$$
\begin{aligned}
\left(\frac{\mathrm{d}}{\mathrm{d} q}-E\right) F_{1}^{\prime}-\frac{F}{2}\left(F_{1}^{\prime}-\frac{F_{2}^{\prime}}{6}\right) & =0 \\
\left(\frac{\mathrm{d}}{\mathrm{d} q}-2 E-\frac{3}{2} F\right)\left(\frac{\mathrm{d}}{\mathrm{d} q}-E\right) F_{2}^{\prime}+\frac{3}{2}\left(2 F^{\prime}-2 E F-F^{2}\right)\left(F_{1}^{\prime}-\frac{F_{2}^{\prime}}{6}\right) & =0
\end{aligned}
$$

where $F_{1}(q)$ and $F_{2}(q)$ are given by

$$
\begin{aligned}
& F_{1}=W^{\prime}+E W-\frac{1}{4}\left(F^{\prime}-2 W F+2 E F+F^{2}\right), \\
& F_{2}=E^{\prime}+E^{2}+\frac{1}{2}\left(F^{\prime}-2 W F+2 E F+F^{2}\right) .
\end{aligned}
$$


We note that the factorized form of the type B 3-fold supercharge component (3.1) is expanded as

$$
P_{3}^{-}=\frac{\mathrm{d}^{3}}{\mathrm{~d} q^{3}}+\sum_{k=0}^{2} w_{k}^{[3]}(q) \frac{\mathrm{d}^{k}}{\mathrm{~d} q^{k}}
$$

with

$$
\begin{aligned}
w_{2}^{[3]}= & 3 W-F, \\
w_{1}^{[3]}= & 3 W^{\prime}+2 E^{\prime}+3 W^{2}-E^{2}-2 W F-E F, \\
w_{0}^{[3]}= & W^{\prime \prime}+E^{\prime \prime}+3 W W^{\prime}+2 E^{\prime} W-E E^{\prime}-W^{\prime} F-E^{\prime} F \\
& +W^{3}-E^{2} W-W^{2} F-E W F .
\end{aligned}
$$

Then, the same conditions (3.4)-(3.7) can be also derived from the general form of 3-fold SUSY in Ref. [11] by substituting (3.9) into the formulas in the latter reference.

To solve the coupled differential equations (3.5) and (3.6), it is convenient to make a change of variable $z=z(q)$ and to introduce a function $f(z)$ defined by

$$
E(q)=\frac{z^{\prime \prime}(q)}{z^{\prime}(q)}, \quad F(q)=\frac{f^{\prime \prime \prime}(z(q))}{f^{\prime \prime}(z(q))} z^{\prime}(q) .
$$

In terms of the new variable $z$ and the function $f(z)$, Eqs. (3.5) and (3.6) are converted into

$$
\begin{array}{r}
\tilde{F}_{1}^{\prime \prime}(z)-\frac{f^{\prime \prime \prime}(z)}{2 f^{\prime \prime}(z)}\left(\tilde{F}_{1}^{\prime}(z)-\frac{\tilde{F}_{2}^{\prime}(z)}{6}\right)=0 \\
\left(\frac{\mathrm{d}}{\mathrm{d} z}-\frac{3 f^{\prime \prime \prime}(z)}{2 f^{\prime \prime}(z)}\right) \tilde{F}_{2}^{\prime \prime}(z)+\frac{3}{2}\left(\frac{2 f^{\prime \prime \prime \prime}(z)}{f^{\prime \prime}(z)}-\frac{3 f^{\prime \prime \prime}(z)^{2}}{f^{\prime \prime}(z)^{2}}\right)\left(\tilde{F}_{1}^{\prime}(z)-\frac{\tilde{F}_{2}^{\prime}(z)}{6}\right)=0
\end{array}
$$

where $\tilde{F}_{i}(z(q))=F_{i}(q)(i=1,2)$. Eliminating $\tilde{F}_{2}(z)$ from (3.11) and (3.12), we obtain

$$
\frac{\mathrm{d}}{\mathrm{d} z}\left(\frac{f^{\prime \prime}(z)}{f^{\prime \prime \prime}(z)} \tilde{F}_{1}^{\prime \prime \prime}(z)-\frac{f^{\prime \prime}(z) f^{\prime \prime \prime \prime}(z)}{f^{\prime \prime \prime}(z)^{2}} \tilde{F}_{1}^{\prime \prime}(z)\right)-\left(\tilde{F}_{1}^{\prime \prime \prime}(z)-\frac{f^{\prime \prime \prime \prime}(z)}{f^{\prime \prime \prime}(z)} \tilde{F}_{1}^{\prime \prime}(z)\right)=0 .
$$

This differential equation for $\tilde{F}_{1}(z)$ can be integrated four times to yield

$$
\tilde{F}_{1}(z)=C_{1}\left(z f^{\prime}(z)-2 f(z)\right)+C_{2} f^{\prime}(z)+C_{3} z+C_{4}
$$

where $C_{i}(i=1, \ldots, 4)$ are integral constants. Substituting it into (3.11) and integrating the resultant differential equation for $\tilde{F}_{2}(z)$, we have

$$
\tilde{F}_{2}(z)=-6\left(C_{1} z f^{\prime}(z)+C_{2} f^{\prime}(z)-C_{3} z-C_{5}\right),
$$

where $C_{5}$ is another integral constant. From the definition (3.10), we can express $z$ and $f(z(q))$ in terms of $E(q)$ and $F(q)$ as

$$
z(q)=\int \mathrm{d} q \mathrm{e}^{\int^{q} \mathrm{~d} q^{\prime} E\left(q^{\prime}\right)}, \quad f(z(q))=\int \mathrm{d} q\left[\mathrm{e}^{\int^{q} \mathrm{~d} q^{\prime} E\left(q^{\prime}\right)} \int^{q} \mathrm{~d} q^{\prime} \mathrm{e}^{\int^{q^{\prime}} \mathrm{d} q^{\prime \prime}\left(E\left(q^{\prime \prime}\right)+F\left(q^{\prime \prime}\right)\right)}\right] .
$$


Substituting them into (3.14) and (3.15), we obtain expressions for $F_{1}(q)$ and $F_{2}(q)$ defined in (3.7). Finally, substituting the resulting $F_{1}(q)$ and $F_{2}(q)$ into (3.7), we obtain the entire relations to be satisfied among the three functions $W(q), E(q)$, and $F(q)$. For instance, the coupled differential equations (3.7) can be integrated for $W(q)$ as

$$
W(q)=\mathrm{e}^{-\int \mathrm{d} q E(q)} \int \mathrm{d} q \mathrm{e}^{\int^{q} \mathrm{~d} q^{\prime} E\left(q^{\prime}\right)}\left(F_{1}(q)+\frac{F_{2}(q)-E^{\prime}(q)-E(q)^{2}}{2}\right) .
$$

Thus, we can express $W(q)$ in terms of $E(q)$ and $F(q)$ by the substitution for the obtained $F_{1}(q)$ and $F_{2}(q)$ into (3.17). Then, eliminating $W(q)$ in (3.7) by using (3.17), we have a relation between $E(q)$ and $F(q)$. In this way, we can obtain the most general form of type B 3-fold SUSY.

Next, we shall show that type B 3-fold SUSY systems are essentially identical with secondorder linear differential operators preserving the space (2.3) investigated in Section II. For this purpose, let us first make a "gauge" transformation on $\mathrm{H}^{-}$:

$$
\tilde{H}^{-}=\mathrm{e}^{\mathcal{W}_{3}^{-}} H^{-} \mathrm{e}^{-\mathcal{W}_{3}^{-}},
$$

with the gauge potential $\mathcal{W}_{3}^{-}(q)$ given by

$$
\mathcal{W}_{3}^{ \pm}(q)=\int \mathrm{d} q(E(q) \mp W(q))
$$

Substituting (3.4) for $V^{-}(q)$ into (3.2), we have

$$
\tilde{H}^{-}=-\frac{1}{2} \frac{\mathrm{d}^{2}}{\mathrm{~d} q^{2}}+(W(q)+E(q)) \frac{\mathrm{d}}{\mathrm{d} q}-F_{1}(q)-\frac{F_{2}(q)}{6},
$$

where $F_{1}(q)$ and $F_{2}(q)$ are given by (3.7). Thus, if we make a change of variable $z=z(q)$ and introduce two functions $A(z)$ and $Q(z)$ as

$$
2 A(z(q))=z^{\prime}(q)^{2}, \quad Q(z(q))=-z^{\prime}(q) W(q)
$$

we can express the gauged Hamiltonian $\tilde{H}^{-}$in terms of $z$ as

$$
\tilde{H}^{-}=-A(z) \frac{\mathrm{d}^{2}}{\mathrm{~d} z^{2}}-\left(Q(z)-\frac{A^{\prime}(z)}{2}\right) \frac{\mathrm{d}}{\mathrm{d} z}-\tilde{F}_{1}(z)-\frac{\tilde{F}_{2}(z)}{6} .
$$

Comparing it with (2.5), we immediately see the correspondence between them as

$$
B(z)=Q(z)-\frac{A^{\prime}(z)}{2}, \quad C(z)=\tilde{F}_{1}(z)+\frac{\tilde{F}_{2}(z)}{6} .
$$

On the other hand, we can convert the formulas among the functions of $q$ in (3.7) into the ones among the functions of $z$ by employing (3.10) and (3.21) as

$$
\begin{aligned}
& \tilde{F}_{1}(z)=-Q^{\prime}(z)-\frac{2 f^{\prime \prime \prime \prime}(z) A(z)+f^{\prime \prime \prime}(z)\left(3 A^{\prime}(z)+2 Q(z)\right)}{4 f^{\prime \prime}(z)}, \\
& \tilde{F}_{2}(z)=A^{\prime \prime}(z)+\frac{2 f^{\prime \prime \prime \prime}(z) A(z)+f^{\prime \prime \prime}(z)\left(3 A^{\prime}(z)+2 Q(z)\right)}{2 f^{\prime \prime}(z)} .
\end{aligned}
$$


Substituting (3.24) into (3.14) and (3.15), we have coupled differential equations for $A(z)$ and $Q(z)$. The latter equations can be easily integrated out to yield

$$
\begin{aligned}
2 B(z)= & 2 Q(z)-A^{\prime}(z) \\
= & 4 C_{1} z f(z)+4 C_{2} f(z)-4 C_{3} z^{2}-2\left(C_{4}+3 C_{5}\right) z-2 C_{6}, \\
A(z) f^{\prime \prime}(z)= & f^{\prime}(z)\left[-2 C_{1} z f(z)-2 C_{2} f(z)+2 C_{3} z^{2}+\left(C_{4}+3 C_{5}\right) z+C_{6}\right] \\
& +2 f(z)\left(C_{1} f(z)-C_{3} z-C_{4}\right)+C_{7} z+C_{8},
\end{aligned}
$$

where $C_{i}(i=6,7,8)$ are additional integral constants. The explicit form of $C(z)$ can be obtained by substituting (3.14) and (3.15) into the second formula in (3.23) as

$$
C(z)=-2 C_{1} f(z)+2 C_{3} z+C_{4}+C_{5} .
$$

Comparing (3.25)-(3.27) with (2.9)-(2.11), we easily see that they are identical with each other and the parameters are related by

$$
\begin{gathered}
c_{2}=-2 C_{1}, \quad c_{1}=2 C_{3}, \quad c_{0}=C_{4}+C_{5}, \quad b_{2}=2 C_{2}, \\
b_{1}=-2 C_{5}, \quad b_{0}=-C_{6}, \quad a_{2}=C_{5}-C_{4}, \quad a_{1}=C_{7}, \quad a_{0}=C_{8} .
\end{gathered}
$$

Therefore, the gauged type B 3-fold SUSY Hamiltonian (3.22) exactly coincides with the most general second-order linear differential operator (2.12), which leaves the linear space (2.3) invariant. The latter fact can be also derived directly by using the type B 3-fold supercharge without recourse to the explicit form of $\tilde{H}^{-}$. In the gauged $z$-space, the component of type B 3-fold supercharge reads as

$$
\tilde{P}_{3}^{-}[z]=\mathrm{e}^{\mathcal{W}_{3}^{-}} P_{3}^{-} \mathrm{e}^{-\mathcal{W}_{3}^{-}}=z^{\prime}(q)^{3}\left(\frac{\mathrm{d}}{\mathrm{d} z}-\frac{f^{\prime \prime \prime}(z)}{f^{\prime \prime}(z)}\right) \frac{\mathrm{d}^{2}}{\mathrm{~d} z^{2}}
$$

and it actually annihilates all the elements of the linear space (2.3). In other words,

$$
\tilde{\mathcal{V}}_{3}^{-}=\operatorname{ker} \tilde{P}_{3}^{-} \text {. }
$$

On the other hand, it follows from the gauge-transformed version of the intertwining relation (3.3) with upper signs, namely, $\tilde{P}_{3}^{-} \tilde{H}^{-}=\tilde{H}^{+} \tilde{P}_{3}^{-}$, that $\tilde{H}^{-} \operatorname{ker} \tilde{P}_{3}^{-} \subset \operatorname{ker} \tilde{P}_{3}^{-}$. Hence, the equality (3.30) indeed means that any gauged type B 3-fold SUSY Hamiltonian $\tilde{H}^{-}$defined by (3.18) with (3.19) preserves the linear space (2.3).

One of the most advantageous and powerful aspects of the framework of $\mathcal{N}$-fold SUSY is that we can construct simultaneously another weakly quasi-solvable Hamiltonian $H^{+}$which is almost isospectral with $H^{-}$. According to Ref. [4], the partner Hamiltonian $\mathrm{H}^{+}$of 3fold SUSY in the physical $q$-space is connected to the gauged $z$-space by another gauge transformation induced by the gauge potential $\mathcal{W}_{3}^{+}(q)$ already defined in (3.19). With the latter gauge transformation, $\mathrm{H}^{+}$is transformed to a second-order linear differential operator $\bar{H}^{+}$of the variable $z$ which must have the following expression:

$$
\begin{aligned}
\bar{H}^{+}= & \mathrm{e}^{\mathcal{W}_{3}^{+}} H^{+} \mathrm{e}^{-\mathcal{W}_{3}^{+}} \\
= & -A(z) \frac{\mathrm{d}^{2}}{\mathrm{~d} z^{2}}+\left(\frac{A^{\prime}(z)}{2}+Q(z)\right) \frac{\mathrm{d}}{\mathrm{d} z}-C(z)-2 Q^{\prime}(z) \\
& +A^{\prime}(z) \tilde{w}_{2}^{[3]}(z)+2 A(z) \tilde{w}_{2}^{[3] \prime}(z),
\end{aligned}
$$


where $A(z)$ and $Q(z)$ are the same functions as the ones in $(3.21), C(z)$ is the zeroth-order term of $\tilde{H}^{-}$in (2.5) and thus is given by the second formula in (3.23) in our type B case, and $\tilde{w}_{2}^{[3]}(z)$ is defined via the expanded form of the gauged 3-fold supercharge component $\tilde{P}_{3}^{-}$under consideration:

$$
\tilde{P}_{3}^{-}=z^{\prime}(q)^{3}\left(\frac{\mathrm{d}^{3}}{\mathrm{~d} z^{3}}+\sum_{k=0}^{2} \tilde{w}_{k}^{[3]}(z) \frac{\mathrm{d}^{k}}{\mathrm{~d} z^{k}}\right) .
$$

Thus, in our type B case (3.29), it is given by

$$
\tilde{w}_{2}^{[3]}(z)=-\frac{f^{\prime \prime \prime}(z)}{f^{\prime \prime}(z)} .
$$

Substituting (3.25)-(3.28) and (3.33) into (3.31), we can express $\bar{H}^{+}$as

$$
\begin{aligned}
\bar{H}^{+}= & -c_{2} K_{8}-c_{1} K_{7}+b_{2} K_{6}+\left(b_{1}-c_{0}\right) K_{5}+b_{0} K_{4} \\
& -\left(a_{2}-c_{0}\right) K_{3}-a_{1} K_{2}-a_{0} K_{1}-c_{0},
\end{aligned}
$$

where the operators $K_{i}(i=1, \ldots, 8)$ are given by

$$
\begin{aligned}
& K_{1}[z]=\frac{1}{f^{\prime \prime}(z)} \frac{\mathrm{d}^{2}}{\mathrm{~d} z^{2}}+\frac{f^{\prime \prime \prime}(z)}{f^{\prime \prime}(z)^{2}} \frac{\mathrm{d}}{\mathrm{d} z}+\frac{f^{\prime \prime}(z) f^{\prime \prime \prime \prime}(z)-f^{\prime \prime \prime}(z)^{2}}{f^{\prime \prime}(z)^{3}}, \\
& K_{2}[z]=z K_{1}[z]-K_{0}[z], \quad K_{3}[z]=f(z) K_{1}[z]-f^{\prime}(z) K_{0}[z]+1, \\
& K_{4}[z]=f^{\prime}(z) K_{1}[z], \quad K_{5}[z]=f^{\prime}(z) K_{2}[z], \quad K_{6}[z]=f^{\prime}(z) K_{3}[z], \\
& K_{7}[z]=\left(z f^{\prime}(z)-f(z)\right) K_{2}[z], \quad K_{8}[z]=\left(z f^{\prime}(z)-f(z)\right) K_{3}[z],
\end{aligned}
$$

and $K_{0}$ is defined by

$$
K_{0}[z]=\frac{1}{f^{\prime \prime}(z)} \frac{\mathrm{d}}{\mathrm{d} z}+\frac{f^{\prime \prime \prime}(z)}{f^{\prime \prime}(z)^{2}}
$$

By applying the formulas (3.7), (3.10), (3.21), (3.23), and (3.33), the gauged partner Hamiltonian $\bar{H}^{+}$in $(3.31)$ is expressed in terms of $q$ as

$$
\begin{aligned}
\bar{H}^{+}= & -\frac{1}{2} \frac{\mathrm{d}^{2}}{\mathrm{~d} q^{2}}+(E-W) \frac{\mathrm{d}}{\mathrm{d} q}+W^{\prime}+E W \\
& -\frac{1}{6}\left(E^{\prime}+E^{2}+5 F^{\prime}+2 W F-2 E F-F^{2}\right) .
\end{aligned}
$$

Then, we can easily check that the potential term in the original Hamiltonian $H^{+}=$ $\mathrm{e}^{-\mathcal{W}_{3}^{+}} \bar{H}^{+} \mathrm{e}^{\mathcal{W}_{3}^{+}}$coincides exactly with $V^{+}(q)$ in (3.4), which was derived from the direct calculation of the intertwining relation (3.3).

The solvable sector $\overline{\mathcal{V}}_{3}^{+}$preserved by $\bar{H}^{+}$is characterized by the gauge-transformed operator of the transposed component $P_{3}^{+}$of type B 3-fold supercharge:

$$
\bar{P}_{3}^{+}=\mathrm{e}^{\mathcal{W}_{3}^{+}} P_{3}^{+} \mathrm{e}^{-\mathcal{W}_{3}^{+}}=-z^{\prime}(q)^{3} \frac{\mathrm{d}^{2}}{\mathrm{~d} z^{2}}\left(\frac{\mathrm{d}}{\mathrm{d} z}+\frac{f^{\prime \prime \prime}(z)}{f^{\prime \prime}(z)}\right) .
$$


In fact, the gauge transformation with $\mathcal{W}_{3}^{+}$of the intertwining relation (3.3) with lower signs, namely, $\bar{P}_{3}^{+} \bar{H}^{+}=\bar{H}^{-} \bar{P}_{3}^{+}$, immediately leads to $\bar{H}^{+}$ker $\bar{P}_{3}^{+} \subset \operatorname{ker} \bar{P}_{3}^{+}$. Hence, we obtain from (3.38):

$$
\overline{\mathcal{V}}_{3}^{+}[z]=\operatorname{ker} \bar{P}_{3}^{+}=\frac{1}{f^{\prime \prime}(z)}\left\langle 1, f^{\prime}(z), z f^{\prime}(z)-f(z)\right\rangle
$$

We can easily check that the eight linearly independent operators $K_{i}(i=1, \ldots, 8)$ in $(3.35)$ indeed preserve the linear space (3.39). It is worth noting that the space $\overline{\mathcal{V}}_{3}^{+}$in (3.39) is related to the one $\tilde{\mathcal{V}}_{3}^{-}$in $(2.3)$ by

$$
\overline{\mathcal{V}}_{3}^{+}[z]=\left.f^{\prime \prime}(z)^{-1} \tilde{\mathcal{V}}_{3}^{-}[w]\right|_{w=f^{\prime}(z), f(w)=z f^{\prime}(z)-f(z)} .
$$

Then, it follows from this relation that a set of eight linearly independent second-order linear differential operators preserving $\overline{\mathcal{V}}_{3}^{+}$can be constructed directly from $J_{i}(i=1, \ldots, 8)$ in $(2.13)$ as

$$
\left.f^{\prime \prime}(z)^{-1} J_{i}[w] f^{\prime \prime}(z)\right|_{w=f^{\prime}(z), f(w)=z f^{\prime}(z)-f(z)} \quad(i=1, \ldots, 8)
$$

Actually, we can check that all the operators $K_{i}(i=1, \ldots, 8)$ in $(3.35)$ are expressed as linear combinations of the ones in (3.41) as

$$
\begin{aligned}
& K_{1}[z]=f^{\prime \prime}(z)^{-1} J_{1}[w] f^{\prime \prime}(z), \quad K_{2}[z]=f^{\prime \prime}(z)^{-1} J_{4}[w] f^{\prime \prime}(z), \\
& K_{3}[z]=f^{\prime \prime}(z)^{-1}\left(J_{5}[w]-J_{3}[w]+1\right) f^{\prime \prime}(z), \quad K_{4}[z]=f^{\prime \prime}(z)^{-1} J_{2}[w] f^{\prime \prime}(z), \\
& K_{5}[z]=f^{\prime \prime}(z)^{-1} J_{5}[w] f^{\prime \prime}(z), \quad K_{6}[z]=f^{\prime \prime}(z)^{-1} J_{7}[w] f^{\prime \prime}(z), \\
& K_{7}[z]=f^{\prime \prime}(z)^{-1} J_{6}[w] f^{\prime \prime}(z), \quad K_{8}[z]=f^{\prime \prime}(z)^{-1} J_{8}[w] f^{\prime \prime}(z),
\end{aligned}
$$

where the substitution $w=f^{\prime}(z)$ and $f(w)=z f^{\prime}(z)-f(z)$ in each operator $J_{i}[w]$ has been understood.

By construction, it is evident that each of the pair Hamiltonians $H^{ \pm}$, which is connected with the gauged ones $\tilde{H}^{-}$and $\bar{H}^{+}$via (3.18) and (3.31), respectively, preserves a threedimensional vector space $\mathcal{V}_{3}^{ \pm}[q]$ defined by

$$
\begin{aligned}
& \mathcal{V}_{3}^{-}[q]=\tilde{\mathcal{V}}_{3}^{-}[z(q)] \mathrm{e}^{-\mathcal{W}_{3}^{-}(q)}, \\
& \mathcal{V}_{3}^{+}[q]=\overline{\mathcal{V}}_{3}^{+}[z(q)] \mathrm{e}^{-\mathcal{W}_{3}^{+}(q)},
\end{aligned}
$$

where $\tilde{\mathcal{V}}_{3}^{-}[z]$ and $\overline{\mathcal{V}}_{3}^{+}[z]$ is respectively given by (2.3) and (3.39). The linear space $\mathcal{V}_{3}^{ \pm}[q]$ is called a solvable sector of $H^{ \pm}$. If it is in addition, a subspace of the linear space in which the corresponding Hamiltonian acts, e.g., $L^{2}(\mathbb{R})$ in a usual quantum mechanical setting, it is quasi-exactly solvable in the space.

\section{EXAMPLES OF TYPE B 3-FOLD SUSY MODELS}

To demonstrate what kind of quantum Hamiltonians can possess type B 3-fold SUSY, we shall exhibit some examples by choosing a particular function for $f(z)$. 
Let us consider type B 3-fold SUSY models realized with the choice $f(z)=\mathrm{e}^{\nu z}$. In the latter choice, the functions $A(z)$ in $(2.9)$ and $Q(z)$ in (3.23) read as

$$
\begin{aligned}
A(z)= & \frac{c_{2}}{\nu} z \mathrm{e}^{\nu z}-\frac{c_{2}+b_{2} \nu}{\nu^{2}} \mathrm{e}^{\nu z}+\frac{c_{1}}{\nu} z^{2}-\frac{c_{1}+\left(b_{1}-c_{0}\right) \nu}{\nu^{2}} z \\
& -\frac{c_{0}-a_{2}+b_{0} \nu}{\nu^{2}}+\frac{a_{1}}{\nu^{2}} z \mathrm{e}^{-\nu z}+\frac{a_{0}}{\nu^{2}} \mathrm{e}^{-\nu z} \\
Q(z)= & -\frac{c_{2}}{2} z \mathrm{e}^{\nu z}+\frac{b_{2}}{2} \mathrm{e}^{\nu z}-c_{1} z^{2}+\frac{c_{1}+\left(b_{1}-c_{0}\right) \nu}{\nu} z \\
& -\frac{c_{1}+\left(b_{1}-c_{0}\right) \nu-2 b_{0} \nu^{2}}{2 \nu^{2}}-\frac{a_{1}}{2 \nu} z \mathrm{e}^{-\nu z}+\frac{a_{1}-a_{0} \nu}{2 \nu^{2}} \mathrm{e}^{-\nu z} .
\end{aligned}
$$

The change of variable from $z$ to $q$ is determined by the first formula in (3.21), but it cannot be integrated analytically in general. Thus, we shall restrict ourselves to considering some particular cases where the change of variable can be performed analytically. In what follows, we shall present the change of variable $z(q)$, the functions $E(q)$ and $F(q)$ in (3.10) and $W(q)$ in (3.21), which altogether characterize a type B model, the gauge factors $\mathcal{W}_{3}^{ \pm}(q)$ in $(3.19)$, the pair of potentials $V^{ \pm}(q)$ in $(3.4)$, and the solvable sectors $\mathcal{V}_{3}^{ \pm}[q]$ in $(3.43)$.

Example 1. $A(z)=2 \alpha z$.

This case is realized by putting $c_{2}=c_{1}=b_{2}=a_{1}=a_{0}=0, a_{2}=c_{0}+b_{0} \nu$, and $c_{0}=b_{1}+2 \alpha \nu$ in (4.1). Then, we obtain the following:

Change of variable: $z(q)=\alpha q^{2}$.

Functions:

$$
E(q)=\frac{1}{q}, \quad F(q)=2 \alpha \nu q, \quad W(q)=\alpha \nu q-\frac{\alpha+b_{0}}{2 \alpha q} .
$$

Gauge factors:

$$
\mathcal{W}_{3}^{ \pm}(q)=\frac{2 \alpha \pm\left(\alpha+b_{0}\right)}{2 \alpha} \ln |q| \mp \frac{\alpha \nu}{2} q^{2}
$$

Potentials:

$$
V^{ \pm}(q)=\frac{\alpha^{2} \nu^{2}}{2} q^{2}+\frac{\left(b_{0}+\alpha \pm 2 \alpha\right)\left(b_{0}+\alpha \pm 4 \alpha\right)}{8 \alpha^{2} q^{2}}-\frac{b_{0}+\alpha \mp 3 \alpha}{6} \nu
$$

Solvable sectors:

$$
\begin{aligned}
& \mathcal{V}_{3}^{-}[q]=\left\langle 1, q^{2}, \mathrm{e}^{\alpha \nu q^{2}}\right\rangle q^{\frac{b_{0}-\alpha}{2 \alpha}} \mathrm{e}^{-\frac{\alpha \nu}{2} q^{2}} \\
& \mathcal{V}_{3}^{+}[q]=\left\langle 1, q^{2}, \mathrm{e}^{-\alpha \nu q^{2}}\right\rangle q^{-\frac{3 \alpha+b_{0}}{2 \alpha}} \mathrm{e}^{\frac{\alpha \nu}{2} q^{2}}
\end{aligned}
$$

Both of the potentials are radial harmonic oscillators, not only quasi-solvable but also solvable, and in particular quite similar to one of the known type $\mathrm{C} \mathcal{N}$-fold SUSY models (with $\mathcal{N}=3$ ), Case 1 in Ref. [4]. However, a precise comparison tells us that they are slightly different from each other in parameter values. 
Example 2. $A(z)=\alpha z^{2} / 2$.

This case is realized by putting $c_{2}=b_{2}=a_{1}=a_{0}=0, a_{2}=c_{0}+b_{0} \nu$, and $c_{1}=\left(c_{0}-b_{1}\right) \nu=$ $\alpha \nu / 2$ in (4.1). Then, we obtain the following:

Change of variable: $z(q)=\mathrm{e}^{\sqrt{\alpha} q}$.

Functions:

$$
E(q)=\sqrt{\alpha}, \quad F(q)=\sqrt{\alpha} \nu \mathrm{e}^{\sqrt{\alpha} q}, \quad W(q)=\frac{\sqrt{\alpha} \nu}{2} \mathrm{e}^{\sqrt{\alpha} q}-\frac{b_{0}}{\sqrt{\alpha}} \mathrm{e}^{-\sqrt{\alpha} q} .
$$

Gauge factors:

$$
\mathcal{W}_{3}^{ \pm}(q)=\sqrt{\alpha} q \mp \frac{\nu}{2} \mathrm{e}^{\sqrt{\alpha} q} \mp \frac{b_{0}}{\alpha} \mathrm{e}^{-\sqrt{\alpha} q}
$$

Potentials:

$$
V^{ \pm}(q)=\frac{\alpha \nu^{2}}{8} \mathrm{e}^{2 \sqrt{\alpha} q}+\frac{\left(b_{0}\right)^{2}}{2 \alpha} \mathrm{e}^{-2 \sqrt{\alpha} q} \pm \frac{\alpha \nu}{4} \mathrm{e}^{\sqrt{\alpha} q} \pm \frac{3 b_{0}}{2} \mathrm{e}^{-\sqrt{\alpha} q}+\frac{2 \alpha-b_{0} \nu}{6} .
$$

Solvable sectors:

$$
\begin{aligned}
& \mathcal{V}_{3}^{-}[q]=\left\langle 1, \mathrm{e}^{\sqrt{\alpha} q}, \mathrm{e}^{\nu \mathrm{e}^{\sqrt{\alpha} q}}\right\rangle \exp \left(-\sqrt{\alpha} q-\frac{\nu}{2} \mathrm{e}^{\sqrt{\alpha} q}-\frac{b_{0}}{\alpha} \mathrm{e}^{-\sqrt{\alpha} q}\right), \\
& \mathcal{V}_{3}^{+}[q]=\left\langle 1, \mathrm{e}^{\sqrt{\alpha} q}, \mathrm{e}^{-\nu \mathrm{e}^{\sqrt{\alpha} q}}\right\rangle \exp \left(-\sqrt{\alpha} q+\frac{\nu}{2} \mathrm{e}^{\sqrt{\alpha} q}+\frac{b_{0}}{\alpha} \mathrm{e}^{-\sqrt{\alpha} q}\right) .
\end{aligned}
$$

This model quite resembles one of the type A $\mathcal{N}$-fold SUSY systems (with $\mathcal{N}=3$ ), Case III in Ref. [9], which is a quasi-solvable generalization of Morse potential, but actually has a slight difference between them in the precise potential form. The solvable sectors are also different.

Example 3. $A(z)=\left(\alpha \mathrm{e}^{\frac{\nu}{2} z}+\beta \mathrm{e}^{-\frac{\nu}{2} z}\right)^{2} / 2$.

This case is realized by putting $c_{2}=c_{1}=a_{1}=0, c_{0}=b_{1},\left(b_{0} \nu+c_{0}-a_{2}\right)^{2}=-4 a_{0} b_{2} \nu$, $2 b_{2}=-\alpha^{2} \nu$, and $2 a_{0}=\beta^{2} \nu^{2}$ in (4.1). For convenience, we assume that $\alpha \beta>0$. Then, we obtain the following:

Change of variable: $z(q)=\frac{2}{\nu} \ln \tan \frac{\sqrt{\alpha \beta} \nu}{2} q$.

Functions:

$$
\begin{aligned}
& E(q)=-\sqrt{\alpha \beta} \nu \frac{\cos \sqrt{\alpha \beta} \nu q}{\sin \sqrt{\alpha \beta} \nu q}, \quad F(q)=\frac{2 \sqrt{\alpha \beta} \nu}{\sin \sqrt{\alpha \beta} \nu q} \\
& W(q)=\frac{\sqrt{\alpha \beta} \nu}{2 \sin \sqrt{\alpha \beta} \nu q}-\frac{2 b_{0}+\alpha \beta \nu}{4 \sqrt{\alpha \beta}} \sin \sqrt{\alpha \beta} \nu q .
\end{aligned}
$$

Gauge factors:

$$
\mathcal{W}_{3}^{ \pm}(q)=-\ln |\sin \sqrt{\alpha \beta} \nu q| \mp \frac{1}{2} \ln \left|\tan \frac{\sqrt{\alpha \beta} \nu}{2} q\right| \mp \frac{2 b_{0}+\alpha \beta \nu}{4 \alpha \beta \nu} \cos \sqrt{\alpha \beta} \nu q .
$$


Potentials:

$$
\begin{aligned}
V^{ \pm}(q)= & \frac{\left(2 b_{0}+\alpha \beta \nu\right)^{2}}{32 \alpha \beta} \sin ^{2} \sqrt{\alpha \beta} \nu q+\frac{\alpha \beta \nu^{2}}{8 \sin ^{2} \sqrt{\alpha \beta} \nu q}+\frac{2 b_{0}-7 \alpha \beta \nu}{24} \nu \\
\mp & \frac{3\left(2 b_{0}+\alpha \beta \nu\right) \nu}{8} \cos \sqrt{\alpha \beta} \nu q \pm \frac{\alpha \beta \nu^{2}}{4} \frac{\cos \sqrt{\alpha \beta} \nu q}{\sin ^{2} \sqrt{\alpha \beta} \nu q} .
\end{aligned}
$$

Solvable sectors:

$$
\begin{aligned}
\mathcal{V}_{3}^{-}[q]= & \left\langle 1, \ln \tan \frac{\sqrt{\alpha \beta} \nu}{2} q, \tan ^{2} \frac{\sqrt{\alpha \beta} \nu}{2} q\right\rangle\left(\sin \frac{\sqrt{\alpha \beta} \nu}{2} q\right)^{1 / 2} \\
& \times\left(\cos \frac{\sqrt{\alpha \beta} \nu}{2} q\right)^{3 / 2} \exp \left(-\frac{2 b_{0}+\alpha \beta \nu}{4 \alpha \beta \nu} \cos \sqrt{\alpha \beta} \nu q\right), \\
\mathcal{V}_{3}^{+}[q]= & \left\langle 1, \ln \tan \frac{\sqrt{\alpha \beta} \nu}{2} q, \tan ^{-2} \frac{\sqrt{\alpha \beta} \nu}{2} q\right\rangle\left(\sin \frac{\sqrt{\alpha \beta} \nu}{2} q\right)^{3 / 2} \\
& \times\left(\cos \frac{\sqrt{\alpha \beta} \nu}{2} q\right)^{1 / 2} \exp \left(\frac{2 b_{0}+\alpha \beta \nu}{4 \alpha \beta \nu} \cos \sqrt{\alpha \beta} \nu q\right) .
\end{aligned}
$$

As in the previous example, this model also resembles one of the type A $\mathcal{N}$-fold SUSY systems (with $\mathcal{N}=3$ ), Case IV in Ref. [9], which is a quasi-solvable generalization of Pöschl-Teller potential, if the trigonometric functions in the above are properly replaced by hyperbolic ones. See also Ref. [16]. But the element $\ln \tan \sqrt{\alpha \beta} \nu q / 2$ in the solvable sectors clearly shows the peculiarity of type B, which does not appear in any type A system.

\section{TYPE A, B, AND C 3-FOLD SUSY WITH MONOMIAL SUBSPACES}

In this section, we shall examine some particular cases of the most general type B 3-fold SUSY investigated in Section III. Virtually all the $\mathcal{N}$-fold SUSY models (including the ones called with other terminologies) so far constructed in the literature are of the types which preserve a monomial or polynomial subspace. The latter types of second-order linear differential operators for the monomial cases were first classified in Ref. [17]. According to it and to the later reconsiderations in Refs. [4, 5], there are essentially three inequivalent monomial subspaces, except for a few low-dimensional ones, preserved by second-order linear differential operators. They are respectively called type $\mathrm{A}$, type $\mathrm{B}$, and type $\mathrm{C}$ monomial subspaces $[4,9] . \mathcal{N}$-fold SUSY systems associated with them were investigated in general fashions in Refs. [2, 3, 18] for type A, in Ref. [5] for type B, and in Ref. [4] for type C monomial subspaces.

As we have shown in Section III, any second-order linear differential operator which preserves a three-dimensional linear space of functions admitting an analytic expression has type B 3-fold SUSY. It means that all the aforementioned systems which preserve a monomial or polynomial subspace must have type B 3 -fold SUSY too when $\mathcal{N}=3$. Hence, it is interesting to know how those systems in the literature would be realized in the most general type B 3-fold SUSY obtained in Section III.

Let us first begin with type $\mathrm{C}$, since, as we will see later, type A and type B can be

regarded as special cases of type C. A type C monomial subspace $\tilde{\mathcal{V}}_{\mathcal{N}_{1}, \mathcal{N}_{2}}^{(\mathrm{C})}$ of dimension $\mathcal{N}$ is 
defined by $[4]$

$$
\tilde{\mathcal{V}}_{\mathcal{N}_{1}, \mathcal{N}_{2}}^{(\mathrm{C})}=\left\langle 1, z, \ldots, z^{\mathcal{N}_{1}-1}, z^{\lambda}, z^{\lambda+1}, \ldots, z^{\lambda+\mathcal{N}_{2}-1}\right\rangle, \quad \mathcal{N}=\mathcal{N}_{1}+\mathcal{N}_{2} \geq 3
$$

where $\mathcal{N}_{1}$ and $\mathcal{N}_{2}$ are positive integers and $\lambda$ is a real number. The linear space (2.3) we have considered reduces to a type $\mathrm{C}$ monomial subspace of dimension 3 with $\mathcal{N}_{1}=2$ and $\mathcal{N}_{2}=1$ when $f(z)=z^{\lambda}$ :

$$
\left.\tilde{\mathcal{V}}_{3}^{-}\right|_{f(z)=z^{\lambda}}=\left\langle 1, z, z^{\lambda}\right\rangle=\tilde{\mathcal{V}}_{2,1}^{(\mathrm{C})} \quad(\lambda \neq-2,-1, \ldots, 3)
$$

The corresponding gauged 3-fold supercharge component (3.29) which annihilates this space (5.2) reads as

$$
\tilde{P}_{3}^{-}=z^{\prime}(q)^{3}\left(\frac{\mathrm{d}^{2}}{\mathrm{~d} z^{2}}-\frac{\lambda-2}{z}\right) \frac{\mathrm{d}^{2}}{\mathrm{~d} z^{2}}, \quad \tilde{w}_{2}^{[3]}(z)=-\frac{\lambda-2}{z},
$$

where $\tilde{w}_{2}^{[3]}(z)$ is calculated with (3.33). The set of eight second-order linear differential operators (2.13) which preserve the three-dimensional type $\mathrm{C}$ monomial subspace (5.2) is

$$
\begin{aligned}
& \lambda(\lambda-1) J_{1}:=\tilde{J}_{1}(\lambda)=z^{2-\lambda} \frac{\mathrm{d}^{2}}{\mathrm{~d} z^{2}}, \quad \lambda(\lambda-1) J_{2}:=\tilde{J}_{2}(\lambda)=z^{3-\lambda} \frac{\mathrm{d}^{2}}{\mathrm{~d} z^{2}}, \\
& \lambda(\lambda-1) J_{3}:=\tilde{J}_{3}(\lambda)=z^{2} \frac{\mathrm{d}^{2}}{\mathrm{~d} z^{2}}, \quad(\lambda-1) J_{4}:=\tilde{J}_{4}(\lambda)=z \frac{\mathrm{d}^{2}}{\mathrm{~d} z^{2}}-(\lambda-1) \frac{\mathrm{d}}{\mathrm{d} z}, \\
& (\lambda-1) J_{5}:=\tilde{J}_{5}(\lambda)=z^{2} \frac{\mathrm{d}^{2}}{\mathrm{~d} z^{2}}-(\lambda-1) z \frac{\mathrm{d}}{\mathrm{d} z}, \\
& (\lambda-1) J_{6}:=\tilde{J}_{6}(\lambda)=z^{\lambda+1} \frac{\mathrm{d}^{2}}{\mathrm{~d} z^{2}}-(\lambda-1) z^{\lambda} \frac{\mathrm{d}}{\mathrm{d} z}, \\
& \lambda J_{7}:=\tilde{J}_{7}(\lambda)=z^{3} \frac{\mathrm{d}^{2}}{\mathrm{~d} z^{2}}-\lambda z^{2} \frac{\mathrm{d}}{\mathrm{d} z}+\lambda z, \\
& \lambda J_{8}:=\tilde{J}_{8}(\lambda)=z^{\lambda+2} \frac{\mathrm{d}^{2}}{\mathrm{~d} z^{2}}-\lambda z^{\lambda+1} \frac{\mathrm{d}}{\mathrm{d} z}+\lambda z^{\lambda} .
\end{aligned}
$$

On the other hand, in the existing literature there are six linearly independent linear differential operators of at most second order preserving the linear space (5.2), which are summarized in (A1) and (A2). The correspondence between (5.4) and the latter is as follows:

$$
\begin{aligned}
(\lambda-1) J_{0}^{(\mathrm{C})}(\lambda)=\tilde{J}_{3}(\lambda)-\tilde{J}_{5}(\lambda), \quad J_{0-}^{(\mathrm{C})}(\lambda)=\tilde{J}_{4}(\lambda), \quad J_{00}^{(\mathrm{C})}(\lambda)=\tilde{J}_{3}(\lambda), \\
J_{+0}^{(\mathrm{C})}(\lambda)=\tilde{J}_{7}(\lambda), \quad J_{\#-}^{(\mathrm{C})}(\lambda)=\tilde{J}_{6}(\lambda), \quad J_{\# 0}^{(\mathrm{C})}(\lambda)=\tilde{J}_{8}(\lambda) .
\end{aligned}
$$

Hence, we see that the two operators $\tilde{J}_{1}(\lambda)$ and $\tilde{J}_{2}(\lambda)$ have been missed so far in the literature. By using (5.4) and (5.5), the most general second-order linear differential operator (2.12) having an invariant type $\mathrm{C}$ monomial subspace (5.2) is expressed as

$$
\begin{aligned}
\tilde{H}^{-}= & -\frac{c_{2}}{\lambda} \tilde{J}_{8}(\lambda)+\frac{b_{2}}{\lambda-1} \tilde{J}_{6}(\lambda)-\frac{a_{1}}{\lambda(\lambda-1)} \tilde{J}_{2}(\lambda)-\frac{a_{0}}{\lambda(\lambda-1)} \tilde{J}_{1}(\lambda) \\
& -\tilde{a}_{3} J_{+0}^{(\mathrm{C})}(\lambda)-\tilde{a}_{2} J_{00}^{(\mathrm{C})}(\lambda)-\tilde{a}_{1} J_{0-}^{(\mathrm{C})}(\lambda)-\tilde{b}_{0} J_{0}^{(\mathrm{C})}(\lambda)-c_{0},
\end{aligned}
$$


where

$$
\tilde{a}_{3}=\frac{c_{1}}{\lambda}, \quad \tilde{a}_{2}=-\frac{b_{1}-c_{0}}{\lambda-1}+\frac{a_{2}-c_{0}}{\lambda(\lambda-1)}, \quad \tilde{a}_{1}=-\frac{b_{0}}{\lambda-1}, \quad \tilde{b}_{0}=b_{1}-c_{0} .
$$

When we put $c_{2}=b_{2}=a_{1}=a_{0}=0$, it reduces to the gauged type C 3-fold SUSY Hamiltonian constructed in Eq. (3.8) of Ref. [4].

The gauged 3-fold superchage component (3.38) and the solvable sector (3.39) annihilated by it for the plus component in this case read as

$$
\bar{P}_{3}^{+}=-z^{\prime}(q)^{3} \frac{\mathrm{d}^{2}}{\mathrm{~d} z^{2}}\left(\frac{\mathrm{d}}{\mathrm{d} z}+\frac{\lambda-2}{z}\right), \quad \overline{\mathcal{V}}_{3}^{+}=z\left\langle 1, z, z^{1-\lambda}\right\rangle .
$$

The set of eight linearly independent second-order linear differential operators (3.35) which preserve the latter space reduces to

$$
\begin{aligned}
& \lambda(\lambda-1) K_{1}:=\tilde{K}_{1}(\lambda)=z^{2-\lambda} \frac{\mathrm{d}^{2}}{\mathrm{~d} z^{2}}+(\lambda-2) z^{1-\lambda} \frac{\mathrm{d}}{\mathrm{d} z}-(\lambda-2) z^{-\lambda}, \\
& \lambda(\lambda-1) K_{2}:=\tilde{K}_{2}(\lambda)=z^{3-\lambda} \frac{\mathrm{d}^{2}}{\mathrm{~d} z^{2}}+(\lambda-3) z^{2-\lambda} \frac{\mathrm{d}}{\mathrm{d} z}-2(\lambda-2) z^{1-\lambda}, \\
& \lambda(\lambda-1) K_{3}:=\tilde{K}_{3}(\lambda)=z^{2} \frac{\mathrm{d}^{2}}{\mathrm{~d} z^{2}}-2 z \frac{\mathrm{d}}{\mathrm{d} z}+2, \\
& (\lambda-1) K_{4}:=\tilde{K}_{4}(\lambda)=z \frac{\mathrm{d}^{2}}{\mathrm{~d} z^{2}}+(\lambda-2) \frac{\mathrm{d}}{\mathrm{d} z}-(\lambda-2) z^{-1}, \\
& (\lambda-1) K_{5}:=\tilde{K}_{5}(\lambda)=z^{2} \frac{\mathrm{d}^{2}}{\mathrm{~d} z^{2}}+(\lambda-3) z \frac{\mathrm{d}}{\mathrm{d} z}-2(\lambda-2), \\
& (\lambda-1) K_{6}:=\tilde{K}_{6}(\lambda)=z^{\lambda+1} \frac{\mathrm{d}^{2}}{\mathrm{~d} z^{2}}-2 z^{\lambda} \frac{\mathrm{d}}{\mathrm{d} z}+2 z^{\lambda-1}, \\
& \lambda K_{7}:=\tilde{K}_{7}(\lambda)=z^{3} \frac{\mathrm{d}^{2}}{\mathrm{~d} z^{2}}+(\lambda-3) z^{2} \frac{\mathrm{d}}{\mathrm{d} z}-2(\lambda-2) z \\
& \lambda K_{8}:=\tilde{K}_{8}(\lambda)=z^{\lambda+2} \frac{\mathrm{d}^{2}}{\mathrm{~d} z^{2}}-2 z^{\lambda+1} \frac{\mathrm{d}}{\mathrm{d} z}+2 z^{\lambda} .
\end{aligned}
$$

The relation to the known quasi-solvable operators listed in (A3) is as follows:

$$
\begin{aligned}
(\lambda-1) K_{0}^{(\mathrm{C})}(\lambda)=\tilde{K}_{5}(\lambda)-\tilde{K}_{3}(\lambda)+\lambda-1, & K_{0-}^{(\mathrm{C})}(\lambda)=\tilde{K}_{4}(\lambda), \\
K_{00}^{(\mathrm{C})}(\lambda)=\tilde{K}_{3}(\lambda), \quad K_{+0}^{(\mathrm{C})}(\lambda)=\tilde{K}_{7}(\lambda), \quad K_{\#-}^{(\mathrm{C})}(\lambda)=\tilde{K}_{1}(\lambda), & K_{\# 0}^{(\mathrm{C})}(\lambda)=\tilde{K}_{2}(\lambda) .
\end{aligned}
$$

Hence, we see again that the two operators $\tilde{K}_{6}(\lambda)$ and $\tilde{K}_{8}(\lambda)$ have been missed in the literature.

Finally, we note that the function $F(q)$ defined in (3.10) is calculated in this case as

$$
F(q)=\frac{(\lambda-2) z^{\prime}(q)}{z(q)}
$$

and coincides (up to a multiplicative factor) with the ad hoc constraint $F(q)=z^{\prime}(q) / z(q)$ made in Ref. [4], Eq. (3.17). We now understand that it naturally comes from the relation (3.10) for the general case of an arbitrary $f(z)$. 
Let us next consider the case associated with a type B monomial subspace. An $\mathcal{N}$ dimensional type $\mathrm{B}$ monomial space is defined by $[4,5]$

$$
\tilde{\mathcal{V}}_{\mathcal{N}}^{(\mathrm{B})}=\left\langle 1, z, \ldots, z^{\mathcal{N}-2}, z^{\mathcal{N}}\right\rangle .
$$

For $\mathcal{N}=3$, it can be realized as a special case of the linear space (2.3) with $f(z)=z^{3}$ or as a special case of the type $\mathrm{C}$ monomial space (5.2) with $\lambda=3$ :

$$
\left.\tilde{\mathcal{V}}_{3}^{-}\right|_{f(z)=z^{3}}=\left.\tilde{\mathcal{V}}_{2,1}^{(\mathrm{C})}\right|_{\lambda=3}=\left\langle 1, z, z^{3}\right\rangle=\tilde{\mathcal{V}}_{3}^{(\mathrm{B})} .
$$

The corresponding gauged 3-fold supercharge component (3.29) which annihilates this space (5.13) reads as

$$
\tilde{P}_{3}^{-}=z^{\prime}(q)^{3}\left(\frac{\mathrm{d}}{\mathrm{d} z}-\frac{1}{z}\right) \frac{\mathrm{d}^{2}}{\mathrm{~d} z^{2}}, \quad \tilde{w}_{2}^{[3]}(z)=-\frac{1}{z},
$$

where $\tilde{w}_{2}^{[3]}(z)$ is calculated with (3.33). The set of eight second-order linear differential operators (2.13) which leave the three-dimensional type B monomial subspace (5.13) invariant is

$$
\begin{aligned}
& \tilde{J}_{1}(3)=z^{-1} \frac{\mathrm{d}^{2}}{\mathrm{~d} z^{2}}, \quad \tilde{J}_{2}(3)=\frac{\mathrm{d}^{2}}{\mathrm{~d} z^{2}}, \quad \tilde{J}_{3}(3)=z^{2} \frac{\mathrm{d}^{2}}{\mathrm{~d} z^{2}}, \\
& \tilde{J}_{4}(3)=z \frac{\mathrm{d}^{2}}{\mathrm{~d} z^{2}}-2 \frac{\mathrm{d}}{\mathrm{d} z}, \quad \tilde{J}_{5}(3)=z^{2} \frac{\mathrm{d}^{2}}{\mathrm{~d} z^{2}}-2 z \frac{\mathrm{d}}{\mathrm{d} z}, \quad \tilde{J}_{6}(3)=z^{4} \frac{\mathrm{d}^{2}}{\mathrm{~d} z^{2}}-2 z^{3} \frac{\mathrm{d}}{\mathrm{d} z}, \\
& \tilde{J}_{7}(3)=z^{3} \frac{\mathrm{d}^{2}}{\mathrm{~d} z^{2}}-3 z^{2} \frac{\mathrm{d}}{\mathrm{d} z}+3 z, \quad \tilde{J}_{8}(3)=z^{5} \frac{\mathrm{d}^{2}}{\mathrm{~d} z^{2}}-3 z^{4} \frac{\mathrm{d}}{\mathrm{d} z}+3 z^{3} .
\end{aligned}
$$

On the other hand, in the existing literature there are seven linearly independent linear differential operators of at most second order preserving the linear space (5.13), which are summarized in (B1) and (B2). The correspondence between (5.15) and the latter is as follows:

$$
\begin{aligned}
& 2 J_{0}^{(\mathrm{B})}=\tilde{J}_{3}(3)-\tilde{J}_{5}(3), \quad J_{--}^{(\mathrm{B})}=\tilde{J}_{2}(3), \quad J_{0-}^{(\mathrm{B})}=\tilde{J}_{4}(3), \\
& J_{00}^{(\mathrm{B})}=\tilde{J}_{3}(3), \quad J_{+0}^{(\mathrm{B})}=\tilde{J}_{7}(3), \quad J_{++}^{(\mathrm{B})}=\tilde{J}_{6}(3), \quad J_{3+}^{(\mathrm{B})}=\tilde{J}_{8}(3) .
\end{aligned}
$$

Hence, we see that the one operator $\tilde{J}_{1}(3)$ has been missed so far in the literature. By using (5.15) and (5.16), the most general second-order linear differential operator (2.12) having an invariant type B monomial subspace (5.13) is expressed as

$$
\tilde{H}^{-}=-\frac{c_{2}}{3} \tilde{J}_{8}(3)-\frac{a_{0}}{6} \tilde{J}_{1}(3)-\sum_{\substack{i, j=+, 0,-i \geq j}} \tilde{a}_{i j} \tilde{J}_{i j}^{(\mathrm{B})}-\tilde{b}_{0} \tilde{J}_{0}^{(\mathrm{B})}-c_{0},
$$

where

$$
\begin{array}{lll}
2 \tilde{a}_{++}=-b_{2}, & 3 \tilde{a}_{+0}=c_{1}, & 6 \tilde{a}_{00}=a_{2}-3 b_{1}-2 c_{0}, \\
2 \tilde{a}_{0-}=-b_{0}, & 6 \tilde{a}_{--}=a_{1}, & \tilde{b}_{0}=b_{1}-c_{0} .
\end{array}
$$

When we put $c_{2}=a_{0}=0$, it reduces to the gauged type B 3-fold SUSY Hamiltonian in Eq. (3.12) of Ref. [5]. 
The gauged 3-fold superchage component (3.38) and the solvable sector (3.39) annihilated by it for the plus component in this case read as

$$
\bar{P}_{3}^{+}=-z^{\prime}(q)^{3} \frac{\mathrm{d}^{2}}{\mathrm{~d} z^{2}}\left(\frac{\mathrm{d}}{\mathrm{d} z}+\frac{1}{z}\right), \quad \overline{\mathcal{V}}_{3}^{+}=z^{-1}\left\langle 1, z^{2}, z^{3}\right\rangle .
$$

The set of eight linearly independent second-order linear differential operators (3.35) which preserve the latter space reduces to

$$
\begin{aligned}
& \tilde{K}_{1}(3)=z^{-1} \frac{\mathrm{d}^{2}}{\mathrm{~d} z^{2}}+z^{-2} \frac{\mathrm{d}}{\mathrm{d} z}-z^{-3}, \quad \tilde{K}_{2}(3)=\frac{\mathrm{d}^{2}}{\mathrm{~d} z^{2}}-2 z^{-2} \\
& \tilde{K}_{3}(3)=z^{2} \frac{\mathrm{d}^{2}}{\mathrm{~d} z^{2}}-2 z \frac{\mathrm{d}}{\mathrm{d} z}+2, \quad \tilde{K}_{4}(3)=z \frac{\mathrm{d}^{2}}{\mathrm{~d} z^{2}}+\frac{\mathrm{d}}{\mathrm{d} z}-z^{-1} \\
& \tilde{K}_{5}(3)=z^{2} \frac{\mathrm{d}^{2}}{\mathrm{~d} z^{2}}+2, \quad \tilde{K}_{6}(3)=z^{4} \frac{\mathrm{d}^{2}}{\mathrm{~d} z^{2}}-2 z^{3} \frac{\mathrm{d}}{\mathrm{d} z}+2 z^{2} \\
& \tilde{K}_{7}(3)=z^{3} \frac{\mathrm{d}^{2}}{\mathrm{~d} z^{2}}-2 z, \quad \tilde{K}_{8}(3)=z^{5} \frac{\mathrm{d}^{2}}{\mathrm{~d} z^{2}}-2 z^{4} \frac{\mathrm{d}}{\mathrm{d} z}+2 z^{3} .
\end{aligned}
$$

The relation to the known quasi-solvable operators listed in (B3) and (B4) is as follows:

$$
\begin{aligned}
& 2 K_{0}^{(\mathrm{B})}=\tilde{K}_{5}(3)-\tilde{K}_{3}(3), \quad K_{--}^{(\mathrm{B})}=\tilde{K}_{2}(3), \quad K_{0-}^{(\mathrm{B})}=\tilde{K}_{4}(3), \\
& K_{00}^{(\mathrm{B})}=\tilde{K}_{5}(3)-2, \quad K_{+0}^{(\mathrm{B})}=\tilde{K}_{7}(3), \quad K_{++}^{(\mathrm{B})}=\tilde{K}_{6}(3), \quad K_{3+}^{(\mathrm{B})}=\tilde{K}_{8}(3) .
\end{aligned}
$$

Hence, we see again that the one operator $\tilde{K}_{1}(3)$ has been missed in the literature.

Finally, the function $F(q)$ defined in (3.10) is calculated in this case as $F(q)=z^{\prime}(q) / z(q)$, and coincides exactly with the ad hoc constraint on $F(q)$ made in Eq. (3.6) of Ref. [5]. As in the case of type $\mathrm{C}$, it naturally comes from the general relation (3.10).

In the last, we shall consider the case associated with a type A monomial subspace. An $\mathcal{N}$-dimensional type A monomial space is defined by $[2,4]$

$$
\tilde{\mathcal{V}}_{\mathcal{N}}^{(\mathrm{A})}=\left\langle 1, z, \ldots, z^{\mathcal{N}-1}\right\rangle \text {. }
$$

For $\mathcal{N}=3$, it can be realized as a special case of the linear space $(2.3)$ with $f(z)=z^{2}$ or as a special case of the type $\mathrm{C}$ monomial space (5.2) with $\lambda=2$ :

$$
\left.\tilde{\mathcal{V}}_{3}^{-}\right|_{f(z)=z^{2}}=\left.\tilde{\mathcal{V}}_{2,1}^{(\mathrm{C})}\right|_{\lambda=2}=\left\langle 1, z, z^{2}\right\rangle=\tilde{\mathcal{V}}_{3}^{(\mathrm{A})} \text {. }
$$

The corresponding gauged 3 -fold supercharge component (3.29) which annihilates this space (5.23) reads as

$$
\tilde{P}_{3}^{-}=z^{\prime}(q)^{3} \frac{\mathrm{d}^{3}}{\mathrm{~d} z^{3}}, \quad \tilde{w}_{2}^{[3]}(z)=0
$$

where $\tilde{w}_{2}^{[3]}(z)$ is calculated with (3.33). The set of eight second-order linear differential operators (2.13) which preserve the three-dimensional type A monomial subspace (5.23) is

$$
\begin{aligned}
& \tilde{J}_{1}(2)=\frac{\mathrm{d}^{2}}{\mathrm{~d} z^{2}}, \quad \tilde{J}_{2}(2)=z \frac{\mathrm{d}^{2}}{\mathrm{~d} z^{2}} \quad \tilde{J}_{3}(2)=z^{2} \frac{\mathrm{d}^{2}}{\mathrm{~d} z^{2}}, \\
& \tilde{J}_{4}(2)=z \frac{\mathrm{d}^{2}}{\mathrm{~d} z^{2}}-\frac{\mathrm{d}}{\mathrm{d} z}, \quad \tilde{J}_{5}(2)=z^{2} \frac{\mathrm{d}^{2}}{\mathrm{~d} z^{2}}-z \frac{\mathrm{d}}{\mathrm{d} z}, \quad \tilde{J}_{6}(2)=z^{3} \frac{\mathrm{d}^{2}}{\mathrm{~d} z^{2}}-z^{2} \frac{\mathrm{d}}{\mathrm{d} z}, \\
& \tilde{J}_{7}(2)=z^{3} \frac{\mathrm{d}^{2}}{\mathrm{~d} z^{2}}-2 z^{2} \frac{\mathrm{d}}{\mathrm{d} z}+2 z, \quad \tilde{J}_{8}(2)=z^{4} \frac{\mathrm{d}^{2}}{\mathrm{~d} z^{2}}-2 z^{3} \frac{\mathrm{d}}{\mathrm{d} z}+2 z^{2} .
\end{aligned}
$$


In contrast with the previous two cases of type $\mathrm{C}$ and type $\mathrm{B}$, it is entirely equivalent with the set of eight linearly independent linear differential operators of at most second order preserving the linear space (5.23) already appeared in the existing literature, which are summarized in (C1). The correspondence between (5.25) and (C1) is as follows:

$$
\begin{aligned}
& J_{-}^{(\mathrm{A})}=\tilde{J}_{2}(2)-\tilde{J}_{4}(2), \quad J_{0}^{(\mathrm{A})}=\tilde{J}_{3}(2)-\tilde{J}_{5}(2), \quad J_{+}^{(\mathrm{A})}=\tilde{J}_{6}(2)-\tilde{J}_{7}(2), \\
& J_{--}^{(\mathrm{A})}=\tilde{J}_{1}(2), \quad J_{0-}^{(\mathrm{A})}=\tilde{J}_{2}(2), \quad J_{00}^{(\mathrm{A})}=\tilde{J}_{3}(3), \quad J_{+0}^{(\mathrm{A})}=\tilde{J}_{6}(3), \quad J_{++}^{(\mathrm{A})}=\tilde{J}_{8}(3) .
\end{aligned}
$$

By using (5.25) and (5.26), the most general second-order linear differential operator (2.12) having an invariant type A monomial subspace (5.23) is expressed as

$$
\tilde{H}^{-}=-\sum_{\substack{i, j=+, 0,-i \geq j}} \tilde{a}_{i j} J_{i j}^{(\mathrm{A})}+\sum_{i=+, 0,-} \tilde{b}_{i} J_{i}^{(\mathrm{A})}-c_{0},
$$

where

$$
\begin{aligned}
& 2 \tilde{a}_{++}=c_{2}, \quad 2 \tilde{a}_{+0}=-2 b_{2}+c_{1}, \quad 2 \tilde{a}_{00}=a_{2}-2 b_{1}+c_{0}, \\
& 2 \tilde{a}_{0-}=a_{1}-2 b_{0}, \quad 2 \tilde{a}_{--}=a_{0}, \quad 2 \tilde{b}_{+}=c_{1}, \quad \tilde{b}_{0}=-b_{1}+c_{0}, \quad \tilde{b}_{-}=-b_{0} .
\end{aligned}
$$

It exactly coincides with the gauged type A 3-fold Hamiltonian in Eq. (4.22) of Ref. [2], which is originated from the $\mathfrak{s l}(2)$ Lie-algebraic quasi-solvable operators in Ref. [19].

The gauged 3-fold superchage component (3.38) and the solvable sector (3.39) annihilated by it for the plus component in this case read as

$$
\bar{P}_{3}^{+}=-z^{\prime}(q)^{3} \frac{\mathrm{d}^{3}}{\mathrm{~d} z^{3}}, \quad \overline{\mathcal{V}}_{3}^{+}=\left\langle 1, z, z^{2}\right\rangle,
$$

and are entirely identical with the ones for the minus component, (5.23) and (5.24). The set of eight linearly independent second-order linear differential operators (3.35) which preserve the latter space reduces to

$$
\begin{aligned}
& \tilde{K}_{1}(2)=\frac{\mathrm{d}^{2}}{\mathrm{~d} z^{2}}, \quad \tilde{K}_{2}(2)=z \frac{\mathrm{d}^{2}}{\mathrm{~d} z^{2}}-\frac{\mathrm{d}}{\mathrm{d} z}, \quad \tilde{K}_{3}(2)=z^{2} \frac{\mathrm{d}^{2}}{\mathrm{~d} z^{2}}-2 z \frac{\mathrm{d}}{\mathrm{d} z}+2, \\
& \tilde{K}_{4}(2)=z \frac{\mathrm{d}^{2}}{\mathrm{~d} z^{2}}, \quad \tilde{K}_{5}(2)=z^{2} \frac{\mathrm{d}^{2}}{\mathrm{~d} z^{2}}-z \frac{\mathrm{d}}{\mathrm{d} z}, \quad \tilde{K}_{6}(2)=z^{3} \frac{\mathrm{d}^{2}}{\mathrm{~d} z^{2}}-2 z^{2} \frac{\mathrm{d}}{\mathrm{d} z}+2 z, \\
& \tilde{K}_{7}(2)=z^{3} \frac{\mathrm{d}^{2}}{\mathrm{~d} z^{2}}-z^{2} \frac{\mathrm{d}}{\mathrm{d} z}, \quad \tilde{K}_{8}(2)=z^{4} \frac{\mathrm{d}^{2}}{\mathrm{~d} z^{2}}-2 z^{3} \frac{\mathrm{d}}{\mathrm{d} z}+2 z^{2},
\end{aligned}
$$

and is again equivalent with the set of eight linearly independent linear differential operators of at most second order preserving the linear space (5.23) in (C1). The correspondence between $(5.25)$ and $(\mathrm{C} 1)$ is as follows (note that $K_{i}^{(\mathrm{A})}=J_{i}^{(\mathrm{A})}$ and $K_{i j}^{(\mathrm{A})}=J_{i j}^{(\mathrm{A})}(i, j=+, 0,-)$ in type $\mathrm{A})$ :

$$
\begin{aligned}
& K_{-}^{(\mathrm{A})}=\tilde{K}_{4}(2)-\tilde{K}_{2}(2), \quad K_{0}^{(\mathrm{A})}=\tilde{K}_{5}(2)-\tilde{K}_{3}(2)+2, \\
& K_{+}^{(\mathrm{A})}=\tilde{K}_{7}(2)-\tilde{K}_{6}(2), \quad K_{--}^{(\mathrm{A})}=\tilde{K}_{1}(2), \quad K_{0-}^{(\mathrm{A})}=\tilde{K}_{4}(2), \\
& K_{00}^{(\mathrm{A})}=2 \tilde{K}_{5}(2)-\tilde{K}_{3}(2)+2, \quad K_{+0}^{(\mathrm{A})}=\tilde{K}_{7}(2), \quad K_{++}^{(\mathrm{A})}=\tilde{K}_{8}(2) .
\end{aligned}
$$

Finally, the function $F(q)$ defined in (3.10) vanishes in this case, $F(q)=0$, which naturally explains the reason why type A 3-fold SUSY can be characterized only by two functions $E(q)$ and $W(q)$. 


\section{COMMUTATION RELATIONS OF THE OPERATORS}

It is well known that some quasi-solvable operators are constructed by enveloping algebra of a Lie algebra. In fact, the discovery of the underlying enveloping algebra of $\mathfrak{s l}(2)$ in [19] promoted the various Lie-algebraic attempts to construct new quasi-solvable models, see, e.g., [20, 21] and references cited therein. However, recent development has shown that there exist several quasi-solvable systems which are not expressible in terms of a differential operator representation of a particular Lie algebra $[4-6,17,22]$. The partial success of the Lie-algebraic approach relies on the elementary fact that, if two operators $J_{i}$ and $J_{j}(i \neq j)$ preserve a linear space $\tilde{\mathcal{V}}$, so does its commutator:

$$
J_{i} \tilde{\mathcal{V}} \subset \tilde{\mathcal{V}}, J_{j} \tilde{\mathcal{V}} \subset \tilde{\mathcal{V}} \Longrightarrow\left[J_{i}, J_{j}\right] \tilde{\mathcal{V}} \subset \tilde{\mathcal{V}}
$$

Needless to say, however, the commutator $\left[J_{i}, J_{j}\right]$ usually has higher order in derivatives than $J_{i}$ and $J_{j}$, and as a consequence is not necessarily included in the set of quasi-solvable operators which is originally considered. In other words, Lie algebra is in general not closed finitely in a finite set of quasi-solvable operators.

In our present case, each commutation relation of $J_{i}(i=1, \ldots, 8)$ is indeed a linear differential operator of third order and thus cannot be expressed as a linear combination of the second-order $J_{i}$, except for the cases when something particular takes place. For a reference, we list every commutation relation in Appendix E. To see what could happen when our quasi-solvable operators turn to be Lie-algebraic, let us consider the following three linear combinations of the operators $J_{i}$ :

$$
J_{-}=J_{2}+\alpha_{-} J_{4}, \quad J_{0}=J_{3}+\alpha_{0} J_{5}, \quad J_{+}=J_{6}+\alpha_{+} J_{7},
$$

where $\alpha_{i}(i=-, 0,+)$ are constants. Their commutators are calculated as

$$
\begin{aligned}
{\left[J_{-}, J_{0}\right]=} & 2 \frac{z f^{\prime}-f+\alpha_{0} z\left(z f^{\prime \prime}-f^{\prime}\right)-\alpha_{-}\left[f f^{\prime \prime}-\left(f^{\prime}\right)^{2}\right]}{f^{\prime \prime}} \frac{\mathrm{d}}{\mathrm{d} z} J_{1} \\
& +\left(\alpha_{0}+1\right) J_{2}+2 \alpha_{0} \frac{z+\alpha_{-} f^{\prime}}{f^{\prime \prime}} \frac{\mathrm{d}}{\mathrm{d} z} J_{4}-\alpha_{-} \alpha_{0} J_{4} \\
{\left[J_{+}, J_{0}\right]=} & -2 \frac{f^{2} f^{\prime \prime}-f\left(f^{\prime}\right)^{2}+\alpha_{+} z\left[\left(\alpha_{0}+1\right) z f f^{\prime \prime}-z\left(f^{\prime}\right)^{2}+f f^{\prime}\right]}{f^{\prime \prime}} \frac{\mathrm{d}}{\mathrm{d} z} J_{1} \\
& -2 \frac{f f^{\prime}+\alpha_{0}\left(z f^{\prime}-f\right) f^{\prime}-\alpha_{+} \alpha_{0} z\left(z f^{\prime}-f\right)}{f^{\prime \prime}} \frac{\mathrm{d}}{\mathrm{d} z} J_{4}-\left(\alpha_{0}+1\right) J_{6} \\
& -2 \alpha_{+} \frac{f+\alpha_{0} z f^{\prime}}{f^{\prime \prime}} \frac{\mathrm{d}}{\mathrm{d} z} J_{9}+\alpha_{+} \alpha_{0} J_{7}, \\
{\left[J_{+}, J_{-}\right]=} & -2 \frac{f\left(z f^{\prime \prime}-f^{\prime}\right)+\alpha_{+} z\left(z^{2} f^{\prime \prime}-z f^{\prime}+f\right)+\alpha_{+} \alpha_{-} z f f^{\prime \prime}}{f^{\prime \prime}} \frac{\mathrm{d}}{\mathrm{d} z} J_{1} \\
& -\left(\alpha_{+} \alpha_{-}+1\right) J_{3}-2 \frac{\left(z+\alpha_{-} f^{\prime}\right) f^{\prime}}{f^{\prime \prime}} \frac{\mathrm{d}}{\mathrm{d} z} J_{4}+\left(2 \alpha_{+} \alpha_{-}-1\right) z J_{4} \\
& -2 \alpha_{+} \frac{z+\alpha_{-} f^{\prime}}{f^{\prime \prime}} \frac{\mathrm{d}}{\mathrm{d} z} J_{9}+\alpha_{+} \alpha_{-} .
\end{aligned}
$$

Hence, it is necessary, though not sufficient, for them to satisfy

$$
z+\alpha_{-} f^{\prime}(z)=f(z)+\alpha_{0} z f^{\prime}(z)=0
$$


in order that they are of second order. This necessary condition is easily solved as

$$
\alpha_{0}=-\frac{1}{2}, \quad f(z)=-\frac{z^{2}}{2 \alpha_{-}} .
$$

The second formula means that it can happen only in the case of type A. In the latter case, the commutators (6.3)-(6.5) read as

$$
\begin{aligned}
& {\left[J_{-}, J_{0}\right]=\frac{1}{2} J_{2}+\frac{\alpha_{-}}{2} J_{4}} \\
& {\left[J_{+}, J_{0}\right]=\frac{1-\alpha_{+} \alpha_{-}}{2\left(\alpha_{-}\right)^{2}} z^{3}\left(z \frac{\mathrm{d}}{\mathrm{d} z} J_{1}+\alpha_{-} \frac{\mathrm{d}}{\mathrm{d} z} J_{4}\right)-\frac{1}{2} J_{6}-\frac{\alpha_{+}}{2} J_{7},} \\
& {\left[J_{+}, J_{-}\right]=-\left(\alpha_{+} \alpha_{-}+1\right) J_{3}+\left(2 \alpha_{+} \alpha_{-}-1\right) J_{5}+\alpha_{+} \alpha_{-} .}
\end{aligned}
$$

Thus, they are all second-order operators if and only if $\alpha_{+} \alpha_{-}=1$. On the other hand, the three operators $J_{i}(i=-, 0,+)$ under the condition (6.7) have the following form:

$$
J_{-}=-\alpha_{-} \frac{\mathrm{d}}{\mathrm{d} z}, \quad J_{0}=\frac{z}{2} \frac{\mathrm{d}}{\mathrm{d} z}, \quad J_{+}=\frac{\alpha_{+} \alpha_{-}-1}{2 \alpha_{-}} z^{3} \frac{\mathrm{d}^{2}}{\mathrm{~d} z^{2}}-\frac{2 \alpha_{+} \alpha_{-}-1}{2 \alpha_{-}} z^{2} \frac{\mathrm{d}}{\mathrm{d} z}+\alpha_{+} z .
$$

Hence, when the condition $\alpha_{+} \alpha_{-}=1$ is satisified, all the three operators $J_{i}(i=-, 0,+)$ turn to be of first order and their Lie algebra is closed simultaneously:

$$
\left[J_{-}, J_{0}\right]=\frac{1}{2} J_{-}, \quad\left[J_{+}, J_{0}\right]=-\frac{1}{2} J_{+}, \quad\left[J_{+}, J_{-}\right]=-2 J_{0}+1 .
$$

That is exactly what takes place in the Lie-algebraic type A case. Indeed, each of the operator $J_{i}(i=-, 0,+)$ is proportional to the corresponding type A quasi-solvable operator $J_{i}^{(\mathrm{A})}(i=-, 0,+)$ in $(5.26)$ when $\alpha_{+} \alpha_{-}=1$. The above example demonstrates that a finite set of quasi-solvable operators preserving a given linear space rarely closes a finitedimensional Lie algebra. Therefore, we can assert that quasi-solvable operators which can be constructed by enveloping algebra of a Lie algebra are rather exceptional.

\section{TYPE $X_{2}$ 3-FOLD SUSY WITH POLYNOMIAL SUBSPACES}

In a certain problem, it is more convenient to start with a more general three-dimensional linear space of functions in a gauged space than (2.3). Type $X_{2} 3$-fold SUSY [8] is such an example. Hence, we shall first reformulate type B 3-fold SUSY in a more general setting. Instead of the linear space (2.3), we shall consider as an invariant subspace

$$
\tilde{\mathcal{V}}_{3}^{\prime-}[u]=\left\langle\tilde{\varphi}_{1}(u), \tilde{\varphi}_{2}(u), \tilde{\varphi}_{3}(u)\right\rangle
$$

in a gauged $u$-space. The relation to the linear space (2.3) in a gauged $z$-space is obviously

$$
\tilde{\mathcal{V}}_{3}^{\prime-}[u]=\left.\tilde{\varphi}_{1}(u) \tilde{\mathcal{V}}_{3}^{-}[z]\right|_{z=\tilde{\varphi}_{2}(u) / \tilde{\varphi}_{1}(u), f(z)=\tilde{\varphi}_{3}(u) / \tilde{\varphi}_{1}(u)} .
$$

With this relation, we can easily produce the most general type B 3-fold SUSY in the $u$-space associated with the linear space (7.1). Indeed, it follows from (7.2) that the gauged type B 3 -fold supercharge component $\tilde{P}_{3}^{\prime-}[u]$ and the eight linearly independent second-order linear 
differential operators $J_{i}^{\prime}[u](i=1, \ldots, 8)$ which preserve the linear space (7.1) in the gauged $u$-space are constructed from the ones in the $z$-space, namely, $\tilde{P}_{3}^{-}[z]$ in $(3.29)$ and $J_{i}[z]$ in (2.13) via the relations

$$
\begin{aligned}
\tilde{P}_{3}^{\prime-}[u] & =\left.\tilde{\varphi}_{1}(u) \tilde{P}_{3}^{-}[z] \tilde{\varphi}_{1}(u)^{-1}\right|_{z=\tilde{\varphi}_{2}(u) / \tilde{\varphi}_{1}(u)}, \\
J_{i}^{\prime}[u] & =\left.\tilde{\varphi}_{1}(u) J_{i}[z] \tilde{\varphi}_{1}(u)^{-1}\right|_{z=\tilde{\varphi}_{2}(u) / \tilde{\varphi}_{1}(u)}
\end{aligned}
$$

Their explicit forms are respectively given by

$$
\tilde{P}_{3}^{\prime-}[u]=u^{\prime}(q)^{3}\left(\frac{\mathrm{d}}{\mathrm{d} u}+\frac{\tilde{\varphi}_{1}^{\prime}}{\tilde{\varphi}_{1}}+\frac{W_{2,1}^{\prime}}{W_{2,1}}-\frac{W_{31,21}^{\prime}}{W_{31,21}}\right)\left(\frac{\mathrm{d}}{\mathrm{d} u}+\frac{\tilde{\varphi}_{1}^{\prime}}{\tilde{\varphi}_{1}}-\frac{W_{2,1}^{\prime}}{W_{2,1}}\right)\left(\frac{\mathrm{d}}{\mathrm{d} u}-\frac{\tilde{\varphi}_{1}^{\prime}}{\tilde{\varphi}_{1}}\right),
$$

where each of the function $W_{i, j}(u)\left(W_{i j, k l}(u)\right.$, respectively) is the Wronskian of $\tilde{\varphi}_{i}(u)$ and $\tilde{\varphi}_{j}(u)$ (of $W_{i, j}(u)$ and $W_{k, l}(u)$, respectively),

$$
\begin{aligned}
W_{i, j}(u) & =\tilde{\varphi}_{i}^{\prime}(u) \tilde{\varphi}_{j}(u)-\tilde{\varphi}_{i}(u) \tilde{\varphi}_{j}^{\prime}(u), \\
W_{i j, k l}(u) & =W_{i, j}^{\prime}(u) W_{k, l}(u)-W_{i, j}(u) W_{k, l}^{\prime}(u),
\end{aligned}
$$

and by

$$
\begin{aligned}
& J_{1}^{\prime}[u]=\frac{W_{2,1}\left(\tilde{\varphi}_{1}\right)^{2}}{W_{31,21}}\left(\frac{\mathrm{d}^{2}}{\mathrm{~d} u^{2}}-\frac{W_{2,1}^{\prime}}{W_{2,1}} \frac{\mathrm{d}}{\mathrm{d} u}+\frac{W_{2,1}^{\prime} \tilde{\varphi}_{1}^{\prime}-W_{2,1} \tilde{\varphi}_{1}^{\prime \prime}}{W_{2,1} \tilde{\varphi}_{1}}\right), \\
& J_{4}^{\prime}[u]=\frac{W_{3,1}\left(\tilde{\varphi}_{1}\right)^{2}}{W_{31,21}}\left(\frac{\mathrm{d}^{2}}{\mathrm{~d} u^{2}}-\frac{W_{2,1}^{\prime}}{W_{2,1}} \frac{\mathrm{d}}{\mathrm{d} u}+\frac{W_{2,1}^{\prime} \tilde{\varphi}_{1}^{\prime}-W_{2,1} \tilde{\varphi}_{1}^{\prime \prime}}{W_{2,1} \tilde{\varphi}_{1}}\right)-\frac{\left(\tilde{\varphi}_{1}\right)^{2}}{W_{2,1}}\left(\frac{\mathrm{d}}{\mathrm{d} u}-\frac{\tilde{\varphi}_{1}^{\prime}}{\tilde{\varphi}_{1}}\right), \\
& J_{2}^{\prime}[u]=\frac{\tilde{\varphi}_{2}}{\tilde{\varphi}_{1}} J_{1}^{\prime}[u], \quad J_{3}^{\prime}[u]=\frac{\tilde{\varphi}_{3}}{\tilde{\varphi}_{1}} J_{1}^{\prime}[u], \quad J_{5}^{\prime}[u]=\frac{\tilde{\varphi}_{2}}{\tilde{\varphi}_{1}} J_{4}^{\prime}[u], \\
& J_{6}^{\prime}[u]=\frac{\tilde{\varphi}_{3}}{\tilde{\varphi}_{1}} J_{4}^{\prime}[u], \quad J_{7}^{\prime}[u]=\frac{\tilde{\varphi}_{2}}{\tilde{\varphi}_{1}} J_{9}^{\prime}[u], \quad J_{8}^{\prime}[u]=\frac{\tilde{\varphi}_{3}}{\tilde{\varphi}_{1}} J_{9}^{\prime}[u],
\end{aligned}
$$

where $J_{9}^{\prime}[u]$ is defined by

$$
J_{9}^{\prime}[u]=\frac{W_{3,2}\left(\tilde{\varphi}_{1}\right)^{2}}{W_{31,21}}\left(\frac{\mathrm{d}^{2}}{\mathrm{~d} u^{2}}-\frac{W_{2,1}^{\prime}}{W_{2,1}} \frac{\mathrm{d}}{\mathrm{d} u}+\frac{W_{2,1}^{\prime} \tilde{\varphi}_{1}^{\prime}-W_{2,1} \tilde{\varphi}_{1}^{\prime \prime}}{W_{2,1} \tilde{\varphi}_{1}}\right)-\frac{\tilde{\varphi}_{2} \tilde{\varphi}_{1}}{W_{2,1}}\left(\frac{\mathrm{d}}{\mathrm{d} u}-\frac{\tilde{\varphi}_{1}^{\prime}}{\tilde{\varphi}_{1}}\right)+1 .
$$

The plus component of the gauged type B 3-fold supercharge $\bar{P}_{3}^{\prime+}[u]$ and the linear space $\overline{\mathcal{V}}_{3}^{\prime+}[u]$ annihilated by it in the $u$-space are related to the ones in the $z$-space, namely, to $\bar{P}_{3}^{+}[z]$ in $(3.38)$ and to $\overline{\mathcal{V}}_{3}^{+}[z]$ in $(3.39)$, respectively, as

$$
\begin{aligned}
\bar{P}_{3}^{\prime+}[u] & =\left.\tilde{\varphi}_{1}(u)^{3} W_{2,1}(u)^{-2} \bar{P}_{3}^{+}[z] W_{2,1}(u)^{2} \tilde{\varphi}_{1}(u)^{-3}\right|_{z=\tilde{\varphi}_{2}(u) / \tilde{\varphi}_{1}(u)}, \\
\overline{\mathcal{V}}_{3}^{\prime+}[u] & =\left.\tilde{\varphi}_{1}(u)^{3} W_{2,1}(u)^{-2} \overline{\mathcal{V}}_{3}^{+}[z]\right|_{z=\tilde{\varphi}_{2}(u) / \tilde{\varphi}_{1}(u)} .
\end{aligned}
$$

Their explicit forms are respectively given by

$$
\bar{P}_{3}^{\prime+}[u]=-u^{\prime}(q)^{3}\left(\frac{\mathrm{d}}{\mathrm{d} u}+\frac{\tilde{\varphi}_{1}^{\prime}}{\tilde{\varphi}_{1}}\right)\left(\frac{\mathrm{d}}{\mathrm{d} u}-\frac{\tilde{\varphi}_{1}^{\prime}}{\tilde{\varphi}_{1}}+\frac{W_{2,1}^{\prime}}{W_{2,1}}\right)\left(\frac{\mathrm{d}}{\mathrm{d} u}-\frac{\tilde{\varphi}_{1}^{\prime}}{\tilde{\varphi}_{1}}-\frac{W_{2,1}^{\prime}}{W_{2,1}}+\frac{W_{31,21}^{\prime}}{W_{31,21}}\right),
$$

and by

$$
\overline{\mathcal{V}}_{3}^{\prime+}[u]=\frac{\tilde{\varphi}_{1}(u)}{W_{31,21}(u)}\left\langle W_{2,1}(u), W_{3,1}(u), W_{3,2}(u)\right\rangle
$$


The eight linearly independent second-order linear differential operators $K_{i}^{\prime}[u](i=1, \ldots, 8)$ which preserve the latter space (7.11) are also constructed from $K_{i}[z]$ in (3.35) via the relation

$$
K_{i}^{\prime}[u]=\left.\tilde{\varphi}_{1}(u)^{3} W_{2,1}(u)^{-2} K_{i}[z] W_{2,1}(u)^{2} \tilde{\varphi}_{1}(u)^{-3}\right|_{z=\tilde{\varphi}_{2}(u) / \tilde{\varphi}_{1}(u)},
$$

and are explicitly given by

$$
\begin{aligned}
K_{1}^{\prime}[u]= & \frac{W_{2,1}\left(\tilde{\varphi}_{1}\right)^{2}}{W_{31,21}}\left[\frac{\mathrm{d}^{2}}{\mathrm{~d} u^{2}}-\left(\frac{2 \tilde{\varphi}_{1}^{\prime}}{\tilde{\varphi}_{1}}-\frac{W_{31,21}^{\prime}}{W_{31,21}}\right) \frac{\mathrm{d}}{\mathrm{d} u}-\frac{\tilde{\varphi}_{1}^{\prime \prime}}{\tilde{\varphi}_{1}}-\frac{W_{2,1}^{\prime \prime}}{W_{2,1}}\right. \\
& \left.+\frac{W_{31,21}^{\prime \prime}}{W_{31,21}}+\frac{2\left(\tilde{\varphi}_{1}^{\prime}\right)^{2}}{\left(\tilde{\varphi}_{1}\right)^{2}}-\left(\frac{\tilde{\varphi}_{1}^{\prime}}{\tilde{\varphi}_{1}}-\frac{W_{2,1}^{\prime}}{W_{2,1}}+\frac{W_{31,21}^{\prime}}{W_{31,21}}\right) \frac{W_{31,21}^{\prime}}{W_{31,21}}\right], \\
K_{2}^{\prime}[u]= & \frac{\tilde{\varphi}_{2}}{\tilde{\varphi}_{1}} K_{1}^{\prime}[u]-K_{0}^{\prime}[u], \quad K_{3}^{\prime}[u]=\frac{\tilde{\varphi}_{3}}{\tilde{\varphi}_{1}} K_{1}^{\prime}[u]-\frac{W_{3,1}}{W_{2,1}} K_{0}^{\prime}[u]+1, \\
K_{4}^{\prime}[u]= & \frac{W_{3,1}}{W_{2,1}} K_{1}^{\prime}[u], \quad K_{5}^{\prime}[u]=\frac{W_{3,1}}{W_{2,1}} K_{2}^{\prime}[u], \quad K_{6}^{\prime}[u]=\frac{W_{3,1}}{W_{2,1}} K_{3}^{\prime}[u], \\
K_{7}^{\prime}[u]= & \frac{W_{3,2}}{W_{2,1}} K_{2}^{\prime}[u], \quad K_{8}^{\prime}[u]=\frac{W_{3,2}}{W_{2,1}} K_{3}^{\prime}[u],
\end{aligned}
$$

where $K_{0}^{\prime}$ is defined by

$$
K_{0}^{\prime}[u]=\frac{\left(W_{2,1}\right)^{2}}{W_{31,21}}\left(\frac{\mathrm{d}}{\mathrm{d} u}-\frac{\tilde{\varphi}_{1}^{\prime}}{\tilde{\varphi}_{1}}-\frac{W_{2,1}^{\prime}}{W_{2,1}}+\frac{W_{31,21}^{\prime}}{W_{31,21}}\right) .
$$

With these preliminaries, let us construct the most general type $X_{2}$ 3-fold SUSY system. The three-dimensional type $X_{2}$ polynomial subspace is introduced as $(7.1)$ with $\tilde{\varphi}_{n}(u)(n=$ $1,2,3)$ given by $[8]$

$$
\tilde{\varphi}_{n}(u ; \alpha)=(\alpha+n-2) u^{n+1}+2(\alpha+n-1)(\alpha-1) u^{n}+(\alpha+n)(\alpha-1) \alpha u^{n-1},
$$

where $\alpha$ is a free parameter. The gauged type $X_{2}$ 3-fold supercharge component is calculated by using the formula (7.4) as

$$
\tilde{P}_{3}^{\prime-}[u ; \alpha]=u^{\prime}(q)^{3} \frac{f_{\alpha}}{f_{\alpha+2}}\left(\frac{\mathrm{d}}{\mathrm{d} u}-\frac{f_{\alpha+3}^{\prime}}{f_{\alpha+3}}\right) \frac{f_{\alpha+2}}{f_{\alpha+1}}\left(\frac{\mathrm{d}}{\mathrm{d} u}-\frac{f_{\alpha+2}^{\prime}}{f_{\alpha+2}}\right) \frac{f_{\alpha+1}}{f_{\alpha}}\left(\frac{\mathrm{d}}{\mathrm{d} u}-\frac{f_{\alpha+1}^{\prime}}{f_{\alpha+1}}\right),
$$

where the function $f_{\alpha}(u)$ is given by

$$
f_{\alpha}(u)=f(u ; \alpha)=u^{2}+2(\alpha-1) u+(\alpha-1) \alpha .
$$

It exactly coincides with Eq. (3.6) for $\mathcal{N}=3$ in Ref. [8]. However, the latter reference reported only four linearly independent second-order linear differential operators $J_{i}^{\left(X_{2}\right)}(\alpha)$ $(i=1, \ldots, 4)$, summarized in (D1) and (D2), which left the type $X_{2}$ polynomial subspace (7.1) with (7.15) invariant. A direct calculation of the eight operators $J_{i}^{\prime}[u]$ in (7.7) using (7.15) shows that the former are expressible as linear combinations of the latter as

$$
J_{i}^{\left(X_{2}\right)}(\alpha)=\sum_{j=1}^{8} C_{i j}(\alpha) J_{j}^{\prime}[u ; \alpha]+C_{i 0}(\alpha) \quad(i=1, \ldots, 4),
$$


where $C_{i j}(\alpha)(i=1, \ldots, 4 ; j=0, \ldots, 8)$ are all constants whose explicit forms are given in Appendix F. Hence, there are four other linear combinations of $J_{i}^{\prime}[u ; \alpha](i=1, \ldots, 8)$ which are linearly independent of $J_{i}^{\left(X_{2}\right)}(\alpha)(i=1, \ldots, 4)$.

The plus component of the gauged type $X_{2}$ 3-fold supercharge $\bar{P}_{3}^{\prime+}$ is similarly calculated from the formula (7.10) with (7.15) as

$$
\bar{P}_{3}^{\prime+}[u]=u^{\prime}(q)^{3}\left(\frac{\mathrm{d}}{\mathrm{d} u}+\frac{f_{\alpha+1}^{\prime}}{f_{\alpha+1}}\right) \frac{f_{\alpha+1}}{f_{\alpha}}\left(\frac{\mathrm{d}}{\mathrm{d} u}+\frac{f_{\alpha+2}^{\prime}}{f_{\alpha+2}}\right) \frac{f_{\alpha+2}}{f_{\alpha+1}}\left(\frac{\mathrm{d}}{\mathrm{d} u}+\frac{f_{\alpha+3}^{\prime}}{f_{\alpha+3}}\right) \frac{f_{\alpha}}{f_{\alpha+2}}
$$

which exactly coincides with Eq. (3.18) for $\mathcal{N}=3$ in Ref. [8]. The linear space $\overline{\mathcal{V}}_{3}^{\prime+}[u](7.11)$ annihilated by it reads by using (7.15) as

$$
\begin{aligned}
\overline{\mathcal{V}}_{3}^{\prime+}[u ; \alpha+3] & =f_{\alpha}(u)^{-1} f_{\alpha+3}(u)^{-1}\left\langle\bar{\chi}_{1}(u, \alpha+3), \bar{\chi}_{2}(u, \alpha+3), \bar{\chi}_{3}(u, \alpha+3)\right\rangle \\
& =f_{\alpha}(u)^{-1} f_{\alpha+3}(u)^{-1} \tilde{\mathcal{V}}_{3}^{\left(X_{2 b}\right)}[u ; \alpha+3],
\end{aligned}
$$

where the function $\bar{\chi}_{n}(u ; \alpha)(n=1,2,3)$ is defined by

$$
\begin{aligned}
\bar{\chi}_{n}(u ; \alpha)= & (\alpha-n)(\alpha-n+1) u^{n+1}+2(\alpha-n-1)(\alpha-n+1)(\alpha-1) u^{n} \\
& +(\alpha-n-1)(\alpha-n)(\alpha-1) \alpha u^{n-1},
\end{aligned}
$$

and thus reproduces Eq. (3.20) for $\mathcal{N}=3$ in Ref. [8]. It is evident that there are eight linearly independent second-order linear differential operators $\tilde{K}_{i}^{\prime}[u ; \alpha+3]$ which preserve the polynomial subspace $\tilde{\mathcal{V}}_{3}^{\left(X_{2 b}\right)}[u ; \alpha+3]$ appeared in (7.20). Since $K_{i}^{\prime}[u](i=1, \ldots, 8)$ in (7.13) leave the linear space $\overline{\mathcal{V}}_{3}^{\prime+}[u ; \alpha+3]$ invariant, it follows from (7.20) that they are given by

$$
\tilde{K}_{i}^{\prime}[u ; \alpha+3]=f_{\alpha}(u)^{-1} f_{\alpha+3}(u)^{-1} K_{i}^{\prime}[u ; \alpha+3] f_{\alpha+3}(u) f_{\alpha}(u) \quad(i=1, \ldots, 8) .
$$

There were again only four linearly independent second-order linear differential operators $K_{i}^{\left(X_{2}\right)}(\alpha)$ in Ref. [8], summarized in (D3) and (D4), which preserved the linear space $\tilde{\mathcal{V}}_{3}^{\left(X_{2 b}\right)}[u ; \alpha]$. A direct calculation of the eight operators $\tilde{K}_{i}^{\prime}[u ; \alpha+3]$ in (7.22) using (7.13) and (7.15) shows that the former are expressible as linear combinations of the latter as

$$
K_{i}^{\left(X_{2}\right)}(\alpha)=\sum_{j=1}^{8} C_{i j}(\alpha-3) \tilde{K}_{j}^{\prime}[u ; \alpha]+C_{i 0}(\alpha-3) \quad(i=1, \ldots, 4),
$$

where $C_{i j}(\alpha-3)(i=1, \ldots, 4 ; j=0, \ldots, 8)$ are all constants whose explicit forms are also given in Appendix F. Hence, there are four other linear combinations of $\tilde{K}_{i}^{\prime}[u ; \alpha]$ $(i=1, \ldots, 8)$ which are linearly independent of $K_{i}^{\left(X_{2}\right)}(\alpha)(i=1, \ldots, 4)$.

\section{DISCUSSION AND SUMMARY}

In this paper, we have shown that type B 3-fold SUSY is a necessary and sufficient condition for a Schrödinger operator to admit three linearly independent local analytic solutions, and thus to preserve a three-dimensional non-polynomial linear space. The most general operator of this kind consists of eight linearly independent non-trivial differential operators of at most second order. As a by-product of the latter finding, we have found 
that all the known 3-fold SUSY associated with monomial or polynomial subspaces, which are regarded as particular cases of the most general type B 3-fold SUSY, also admit eight operators for each component Hamiltonian, some of which have been missed in the literature. In particular, the three different types of 3-fold SUSY associated with monomial subspaces, namely, type A, type B, and type C, are connected continuously via a parameter. The results obtained here are full of implications for the future studies, and we would like to close this paper by referring to some of them.

The fact that the number of linearly independent quasi-solvable operators of at most second order preserving a three-dimensional linear function space is always the same casts a natural question: Is it true for any dimensional linear function space? According to the classification of quasi-solvable operators which leave a monomial space invariant done in Ref. [17], there are no extra operators in the cases of type B and $\mathrm{C}$ monomial subspaces (the general case (4) and the special case $\mathrm{C}$ and $\mathrm{D}$ there) except for the three-dimensional and a particular four-dimensional ones. The number of linearly independent non-trivial quasisolvable operators of at most second order preserving a type A monomial subspace (the general case (3) in Ref. [17]), on the other hand, is always eight regardless of the dimension of the subspace. A mathematical theorem (Corollary 2) in Ref. [22] assures the latter, and further states that the number of linearly independent at most second-order linear differential operators preserving a type B monomial space of dimension greater than three is, excluding the trivial constant multiplication operation, six. Hence, it does not seem to be true in the cases of function spaces of more than three dimension.

Let us next observe the problem from a different point of view. A crucial consequence of the fact in the present three-dimensional case is that the eight linearly independent quasisolvable operators preserving monomial spaces of type $\mathrm{A}$, type $\mathrm{B}$, and type $\mathrm{C}$ are connected continuously with each other via one parameter. In this respect, we note that these three different types are connected continuously, at least at the level of monomial subspaces, also in any dimension of more than three. In fact, an $\mathcal{N}$-dimensional type $\mathrm{C}$ monomial subspace (5.1) with $\mathcal{N}_{1}=\mathcal{N}-1$ and $\mathcal{N}_{2}=1$ reads as

$$
\tilde{\mathcal{V}}_{\mathcal{N}-1,1}^{(\mathrm{C})}=\left\langle 1, z, \ldots, z^{\mathcal{N}-2}, z^{\lambda}\right\rangle
$$

Evidently, it reduces to an $\mathcal{N}$-dimensional type B monomial subspace when $\lambda=\mathcal{N}$ and to a type A one when $\lambda=\mathcal{N}-1$. Hence, in contrast with the previous observation, it seems that the latter two types are connected continuously with the particular case of type $\mathrm{C}$ via the parameter $\lambda$ also in any dimension $\mathcal{N}>3$.

If it is indeed the case, the number of linearly independent quasi-solvable operators in the cases of type B and type C must be the same as the one in the type A case, namely, eight. If it is not the case, on the other hand, some irregular phenomena would take place in the limits $\lambda \rightarrow 1$ and 2. It could be superficial, however, arising from the restriction to at most second-order linear differential operators. Actually, if we restrict our consideration to first-order ones in the present $\mathcal{N}=3$ case, they are no longer connected continuously with each other and the number of linearly independent operators varies (one in type B and type $\mathrm{C}$, three in type $\mathrm{A}$, and zero in general non-monomial cases). Hence, it could be the case that the number is invariable for operators of a certain order higher than two in a linear function space of dimension higher than three.

Another intriguing fact is that there is at least one extra quasi-solvable second-order operator for a particular four-dimensional type $\mathrm{C}$ monomial subspace with $\mathcal{N}_{1}=\mathcal{N}_{2}=2$ 
(the special case B in Ref. [17]):

$$
\tilde{\mathcal{V}}_{2,2}^{(\mathrm{C})}=\left\langle 1, z, z^{\lambda}, z^{\lambda+1}\right\rangle .
$$

Hence, we expect that a detailed examination for the four-dimensional case would give us further insights into the issue.

The fact that the most general type B 3-fold SUSY system admits three linearly independent local solutions of non-polynomial type in closed form indicates that there would be a possibly tremendous number of Schrödinger operators having non-polynomial local solutions which have not been found yet. Until now, virtually all the Schrödinger operators known to have local analytic solutions and thus be (quasi-)solvable are those whose solutions are expressible as products of a polynomial in a particular function and a gauge factor (the ground-state wave function when exactly solvable).

As is well-known, $\mathcal{N}$-fold SUSY is equivalent to weak quasi-solvability for one-dimensional Schrödinger operators and hence any of them admitting local solutions in closed form possesses (at least) one type of $\mathcal{N}$-fold SUSY. There have been four types so far found, and all the constructed $\mathcal{N}$-fold SUSY systems are those having polynomial-type local solutions, or equivalently, those preserving a polynomial subspace. It should be noted, however, that except for type A they constitute only subclasses of the most general $\mathcal{N}$-fold SUSY in each type. In the case of type A, it was already proved $[2,3]$ that it is equivalent to the $\mathfrak{s l}(2)$ Lie-algebraic quasi-solvable models [19] preserving a type A monomial space. On the other hand, all the other types of $\mathcal{N}$-fold SUSY systems were constructed under a particular restriction $F(q)=z^{\prime}(q) / z(q)$ which guarantees that a linear function space preserved by the operator under consideration is of monomial or polynomial type. In other words, linear differential operators admitting an invariant subspace of non-polynomial type have not been systematically considered yet.

To explore the area of operators with a non-polynomial invariant subspace, it is now evident that we must tackle a necessary and sufficient condition for other types of $\mathcal{N}$-fold SUSY, or at least examine them under a less restrictive condition than $F(q)=z^{\prime}(q) / z(q)$. The present investigation on the necessary and sufficient condition for type B 3-fold SUSY would definitely provide us the first clue and foothold toward that research direction.

The notion of shape invariance introduced in Ref. [23] is a well-known tool for investigating and constructing an exactly solvable Schrödinger operator. Precisely speaking, it is a sufficient condition for solvability but is neither necessary nor sufficient for exact solvability. Later in Ref. [24], it was generalized to the notion of two- and multi-step shape invariance, keeping the sufficiency for solvability intact. Recently, it was shown [14] that any two-step shape-invariant model can be systematically investigated and constructed in the framework of $\mathcal{N}$-fold SUSY as a particular case of 2 -fold SUSY with an intermediate Hamiltonian developed in Ref. [13]. Now that we appreciate the most general 3-fold SUSY which is quasi-solvable in the strong sense, we are in a position to follow the latter program to uncover the area of three-step shape invariance. In the case of type A 3-fold SUSY, it was already shown [25] that the possible three-step shape-invariant potentials are all reducible, that is, they have ordinal shape invariance as well. Hence, we would be able to see whether there exists an irreducible three-step shape-invariant potential by using the results here. 


\section{Appendix A: Quasi-solvable operators in type C 3-fold SUSY}

In Ref. [17], the set of quasi-solvable operators up to second order preserving the type $\mathrm{C}$ monomial subspace (5.1) was first investigated and four were found for an arbitrary $\mathcal{N}_{1}, \mathcal{N}_{2} \in \mathbb{N}$, cf. the general case (4-a) there. It was also reexamined in Proposition 3 , Ref. [22], which would be applicable only for $\mathcal{N}_{1}+\mathcal{N}_{2}>3$, though it was not stated explicitly there, assumed in the preceding Theorem 2 . In our present case of $\mathcal{N}_{1}=2$ and $\mathcal{N}_{2}=1$, they reduce to (cf. (3.7a)-(3.7d) in Ref. [4])

$$
\begin{array}{ll}
J_{0}^{(\mathrm{C})}(\lambda)=z \frac{\mathrm{d}}{\mathrm{d} z}, & J_{0-}^{(\mathrm{C})}(\lambda)=\frac{\mathrm{d}}{\mathrm{d} z}\left(z \frac{\mathrm{d}}{\mathrm{d} z}-\lambda\right) \\
J_{00}^{(\mathrm{C})}(\lambda)=z^{2} \frac{\mathrm{d}^{2}}{\mathrm{~d} z^{2}}, & J_{+0}^{(\mathrm{C})}(\lambda)=z\left(z \frac{\mathrm{d}}{\mathrm{d} z}-1\right)\left(z \frac{\mathrm{d}}{\mathrm{d} z}-\lambda\right) .
\end{array}
$$

In addition to the above, there exist two extra second-order operators preserving the threedimensional type $\mathrm{C}$ monomial space (5.2). This is because three-dimensional monomial subspaces are special and it can also be regarded as the special case A (a) in Ref. [17]. The two additional operators are given by

$$
J_{\#-}^{(\mathrm{C})}(\lambda)=z^{\lambda} \frac{\mathrm{d}}{\mathrm{d} z}\left(z \frac{\mathrm{d}}{\mathrm{d} z}-\lambda\right), \quad J_{\# 0}^{(\mathrm{C})}(\lambda)=z^{\lambda}\left(z \frac{\mathrm{d}}{\mathrm{d} z}-1\right)\left(z \frac{\mathrm{d}}{\mathrm{d} z}-\lambda\right),
$$

and were not considered in Ref. [4].

The other set of quasi-solvable operators related with the above via 3-fold SUSY preserves the linear space $\overline{\mathcal{V}}_{3}^{+}$in (5.8) which is equivalent up to a similarity transformation to another type $\mathrm{C}$ monomial subspace $\left\langle 1, z, z^{1-\lambda}\right\rangle$. Hence, it is evident that they are given by $K_{0}^{(\mathrm{C})}(\lambda)=$ $z J_{0}^{(\mathrm{C})}(1-\lambda) z^{-1}$ and $K_{i j}^{(\mathrm{C})}(\lambda)=z J_{i j}^{(\mathrm{C})}(1-\lambda) z^{-1}(i, j=+, 0,-)$, cf. (3.29) in Ref. [4]. Explicitly, their forms read as

$$
\begin{aligned}
& K_{0}^{(\mathrm{C})}(\lambda)=z \frac{\mathrm{d}}{\mathrm{d} z}-1, \quad K_{0-}^{(\mathrm{C})}(\lambda)=z^{-1}\left(z \frac{\mathrm{d}}{\mathrm{d} z}-1\right)\left(z \frac{\mathrm{d}}{\mathrm{d} z}-2+\lambda\right), \\
& K_{00}^{(\mathrm{C})}(\lambda)=\left(z \frac{\mathrm{d}}{\mathrm{d} z}-2\right)\left(z \frac{\mathrm{d}}{\mathrm{d} z}-1\right), \quad K_{+0}^{(\mathrm{C})}(\lambda)=z\left(z \frac{\mathrm{d}}{\mathrm{d} z}-2\right)\left(z \frac{\mathrm{d}}{\mathrm{d} z}-2+\lambda\right), \\
& K_{\#-}^{(\mathrm{C})}(\lambda)=z^{-\lambda}\left(z \frac{\mathrm{d}}{\mathrm{d} z}-1\right)\left(z \frac{\mathrm{d}}{\mathrm{d} z}-2+\lambda\right), \\
& K_{\# 0}^{(\mathrm{C})}(\lambda)=z^{1-\lambda}\left(z \frac{\mathrm{d}}{\mathrm{d} z}-2\right)\left(z \frac{\mathrm{d}}{\mathrm{d} z}-2+\lambda\right) .
\end{aligned}
$$

The last two operators were not considered in Ref. [4].

\section{Appendix B: Quasi-solvable operators in type B 3-fold SUSY}

The set of quasi-solvable operators up to second order preserving the type B monomial subspace (5.12) was also investigated in Ref. [17] and six were found for an arbitrary $\mathcal{N} \in \mathbb{N}$.

It was also restudied in Corollary 2 of Ref. [22], which would be applicable only for $\mathcal{N}>3$ 
assumed in the preceding Theorem 2. In the case of $\mathcal{N}=3$, they reduce to (cf. (3.11a)(3.11f) in Ref. [5])

$$
\begin{aligned}
& J_{0}^{(\mathrm{B})}=z \frac{\mathrm{d}}{\mathrm{d} z}, \quad J_{--}^{(\mathrm{B})}=\frac{\mathrm{d}^{2}}{\mathrm{~d} z^{2}}, \quad J_{0-}^{(\mathrm{B})}=\frac{\mathrm{d}}{\mathrm{d} z}\left(z \frac{\mathrm{d}}{\mathrm{d} z}-3\right), \quad J_{00}^{(\mathrm{B})}=z^{2} \frac{\mathrm{d}^{2}}{\mathrm{~d} z^{2}}, \\
& J_{+0}^{(\mathrm{B})}=z\left(z \frac{\mathrm{d}}{\mathrm{d} z}-3\right)\left(z \frac{\mathrm{d}}{\mathrm{d} z}-1\right), \quad J_{++}^{(\mathrm{B})}=z^{3} \frac{\mathrm{d}}{\mathrm{d} z}\left(z \frac{\mathrm{d}}{\mathrm{d} z}-3\right) .
\end{aligned}
$$

In addition to the above, there exists an extra second-order operator preserving the threedimensional type B monomial space (cf. the special case A (b) in Ref. [17]):

$$
J_{3+}^{(\mathrm{B})}=z^{3}\left(z \frac{\mathrm{d}}{\mathrm{d} z}-3\right)\left(z \frac{\mathrm{d}}{\mathrm{d} z}-1\right) .
$$

The latter operator was not considered in Ref. [5].

The other set of quasi-solvable operators related with the above via 3-fold SUSY, which preserves the linear space $\overline{\mathcal{V}}_{3}^{+}$in (5.19), reads as (cf. (3.16a)-(3.16f) in Ref. [5])

$$
\begin{aligned}
& K_{0}^{(\mathrm{B})}=z \frac{\mathrm{d}}{\mathrm{d} z}, \quad K_{--}^{(\mathrm{B})}=z^{-2}\left(z \frac{\mathrm{d}}{\mathrm{d} z}+1\right)\left(z \frac{\mathrm{d}}{\mathrm{d} z}-2\right), \\
& K_{0-}^{(\mathrm{B})}=z^{-1}\left(z \frac{\mathrm{d}}{\mathrm{d} z}+1\right)\left(z \frac{\mathrm{d}}{\mathrm{d} z}-1\right), \quad K_{00}^{(\mathrm{B})}=z^{2} \frac{\mathrm{d}^{2}}{\mathrm{~d} z^{2}}, \\
& K_{+0}^{(\mathrm{B})}=z\left(z \frac{\mathrm{d}}{\mathrm{d} z}-2\right)\left(z \frac{\mathrm{d}}{\mathrm{d} z}+1\right), \quad K_{++}^{(\mathrm{B})}=z^{2}\left(z \frac{\mathrm{d}}{\mathrm{d} z}-2\right)\left(z \frac{\mathrm{d}}{\mathrm{d} z}-1\right) .
\end{aligned}
$$

The space $\overline{\mathcal{V}}_{3}^{+}$is equivalent to a three-dimensional monomial subspace $\left\langle 1, z^{2}, z^{3}\right\rangle$ up to a similarity transformation. Hence, the latter monomial space is preserved by $z K_{0}^{(\mathrm{B})} z^{-1}$ and $z K_{i j}^{(\mathrm{B})} z^{-1}(i, j=+, 0,-)$. A set of quasi-solvable operators of up to second order preserving it was also examined in Ref. [17] as the special case A (c) with $m=2$ and six were presented. Transformed back to the space $\overline{\mathcal{V}}_{3}^{+}$in $(5.19)$, they correspond to $K_{0}^{(\mathrm{B})}, K_{0-}^{(\mathrm{B})}, K_{00}^{(\mathrm{B})}, K_{+0}^{(\mathrm{B})}$, $K_{++}^{(\mathrm{B})}$, and another one given by

$$
K_{3+}^{(\mathrm{B})}=z^{3}\left(z \frac{\mathrm{d}}{\mathrm{d} z}-2\right)\left(z \frac{\mathrm{d}}{\mathrm{d} z}-1\right) .
$$

Hence, there have been in total seven linearly independent ones so far found in Refs. [5, 17].

\section{Appendix C: Quasi-solvable operators in type A 3-fold SUSY}

The set of quasi-solvable operators up to second order preserving the type A monomial subspace (5.22) is exhausted by the first-order differential operator representation of $\mathfrak{s l}(2)$ Lie algebra and their quadratic form, which was first reported in Ref. [19] (cf. (4.13) and (4.17)-(4.21) in Ref. [2]):

$$
\begin{aligned}
& J_{-}^{(\mathrm{A})}=\frac{\mathrm{d}}{\mathrm{d} z}, \quad J_{0}^{(\mathrm{A})}=z \frac{\mathrm{d}}{\mathrm{d} z}, \quad J_{+}^{(\mathrm{A})}=z\left(z \frac{\mathrm{d}}{\mathrm{d} z}-2\right), \\
& J_{--}^{(\mathrm{A})}=\frac{\mathrm{d}^{2}}{\mathrm{~d} z^{2}}, \quad J_{0-}^{(\mathrm{A})}=z \frac{\mathrm{d}^{2}}{\mathrm{~d} z^{2}}, \quad J_{00}^{(\mathrm{A})}=z^{2} \frac{\mathrm{d}^{2}}{\mathrm{~d} z^{2}}, \\
& J_{+0}^{(\mathrm{A})}=z^{2} \frac{\mathrm{d}}{\mathrm{d} z}\left(z \frac{\mathrm{d}}{\mathrm{d} z}-2\right), \quad J_{++}^{(\mathrm{A})}=z^{2}\left(z \frac{\mathrm{d}}{\mathrm{d} z}-2\right)\left(z \frac{\mathrm{d}}{\mathrm{d} z}-1\right) .
\end{aligned}
$$


The other set of quasi-solvable operators related with the above via 3-fold SUSY preserves the same type A monomial subspace (5.22) and thus is the same, as shown in Ref. [3] for arbitrary $\mathcal{N} \in \mathbb{N}$. Hence, we have $K_{i}^{(\mathrm{A})}=J_{i}^{(\mathrm{A})}$ and $K_{i j}^{(\mathrm{A})}=J_{i j}^{(\mathrm{A})}(i=+, 0,-)$.

\section{Appendix D: Quasi-solvable operators in type $X_{2}$ 3-fold SUSY}

In Ref. [8], second-order quasi-solvable operators preserving a type $X_{2}$ exceptional polynomial subspace were first studied and four linearly independent ones were found for each

subspace. In the case of three-dimensional subspace $\tilde{\mathcal{V}}_{3}^{-{ }^{\prime}}[u ; \alpha]=\tilde{\mathcal{V}}_{3}^{\left(X_{2 a}\right)}[u ; \alpha]$, namely, (7.1) with (7.15), they reduce to (cf. (2.12), (2.15), (2.18), and (2.20) in Ref. [8])

$$
\begin{aligned}
J_{1}^{\left(X_{2}\right)}(\alpha)= & u \frac{\mathrm{d}^{2}}{\mathrm{~d} u^{2}}-(u-\alpha+3) \frac{\mathrm{d}}{\mathrm{d} u}+\frac{4(\alpha-1)(u+\alpha)}{f(u ; \alpha)}\left(\frac{\mathrm{d}}{\mathrm{d} u}-1\right) \\
J_{2}^{\left(X_{2}\right)}(\alpha)= & {\left[u^{2}+(\alpha+2)(\alpha-1)\right] \frac{\mathrm{d}^{2}}{\mathrm{~d} u^{2}}-\left[u^{2}+4 u+(\alpha-1)(3 \alpha+2)\right] \frac{\mathrm{d}}{\mathrm{d} u}+4 u } \\
& -8(\alpha-1) \frac{\alpha u+\alpha^{2}-1}{f(u ; \alpha)}\left(\frac{\mathrm{d}}{\mathrm{d} u}-1\right), \\
J_{3}^{\left(X_{2}\right)}(\alpha)= & (u+2 \alpha+2) u^{2} \frac{\mathrm{d}^{2}}{\mathrm{~d} u^{2}}+\left[(\alpha-5) u^{2}+\left(3 \alpha^{2}+\alpha-8\right) u+4(\alpha+4)(\alpha-1)\right] \frac{\mathrm{d}}{\mathrm{d} u} \\
& -4(\alpha-2) u-4(\alpha-1) \frac{\left(\alpha^{2}+3 \alpha-8\right) u+(\alpha+4)(\alpha-1) \alpha}{f(u ; \alpha)}\left(\frac{\mathrm{d}}{\mathrm{d} u}-1\right),
\end{aligned}
$$

and

$$
\begin{aligned}
2(\alpha+ & 1) J_{4}^{\left(X_{2}\right)}(\alpha)=\left[2(\alpha+1) u+3 \alpha^{2}+7 \alpha+6\right] u^{3} \frac{\mathrm{d}^{2}}{\mathrm{~d} u^{2}}-\left[12(\alpha+1) u^{3}\right. \\
& +\left(3 \alpha^{3}+8 \alpha^{2}+15 \alpha+22\right) u^{2}+(\alpha-1)\left(7 \alpha^{3}+27 \alpha^{2}+10 \alpha-16\right) u \\
& \left.+2(\alpha-1)\left(\alpha^{4}+8 \alpha^{3}+29 \alpha^{2}-6 \alpha-40\right)\right] \frac{\mathrm{d}}{\mathrm{d} u} \\
& +24(\alpha+1) u^{2}+4(\alpha-1)\left(3 \alpha^{2}+2 \alpha-4\right) u \\
& +4(\alpha-1)^{2} \frac{\left(\alpha^{3}+9 \alpha^{2}-22 \alpha-40\right) u+\left(\alpha^{3}+9 \alpha^{2}-6 \alpha-20\right) \alpha}{f(u ; \alpha)}\left(\frac{\mathrm{d}}{\mathrm{d} u}-1\right) .
\end{aligned}
$$

The other set of quasi-solvable operators related with the above via 3-fold SUSY, which preserves the other $X_{2}$ exceptional polynomial subspace $\tilde{\mathcal{V}}_{3}^{\left(X_{2 b}\right)}[u ; \alpha]$ defined in (7.20) with 
(7.21), reads as (cf. (3.38), (3.41), (3.44), and (3.46) in Ref. [8])

$$
\begin{aligned}
K_{1}^{\left(X_{2}\right)}(\alpha)= & u \frac{\mathrm{d}^{2}}{\mathrm{~d} u^{2}}+(u-\alpha-3) \frac{\mathrm{d}}{\mathrm{d} u}+\frac{4}{f(u ; \alpha)}\left[(\alpha-1)(u+\alpha) \frac{\mathrm{d}}{\mathrm{d} u}+\alpha(u+\alpha-1)\right], \\
K_{2}^{\left(X_{2}\right)}(\alpha)= & {\left[u^{2}+(\alpha-1)(\alpha-4)\right] \frac{\mathrm{d}^{2}}{\mathrm{~d} u^{2}}+\left[u^{2}-6 u+(\alpha-1)(3 \alpha-8)\right] \frac{\mathrm{d}}{\mathrm{d} u}-4 u } \\
& -\frac{8(\alpha-1)}{f(u ; \alpha)}\left\{[(\alpha-3) u+(\alpha-1)(\alpha-2)] \frac{\mathrm{d}}{\mathrm{d} u}+(\alpha-2) u+\alpha^{2}-3 \alpha+4\right\}, \\
K_{3}^{\left(X_{2}\right)}(\alpha)= & (u+2 \alpha-4) u^{2} \frac{\mathrm{d}^{2}}{\mathrm{~d} u^{2}}-\left[(\alpha+3) u^{2}+\left(3 \alpha^{2}-5 \alpha-4\right) u-4(\alpha-1)(\alpha-2)\right] \frac{\mathrm{d}}{\mathrm{d} u} \\
& +4 \alpha u-\frac{4(\alpha-1)}{f(u ; \alpha)}\left\{\left[\left(\alpha^{2}-3 \alpha+4\right) u+\alpha(\alpha-1)(\alpha-2)\right] \frac{\mathrm{d}}{\mathrm{d} u}\right. \\
& +\alpha[(\alpha-2) u+\alpha(\alpha-3)]\},
\end{aligned}
$$

and

$$
\begin{aligned}
& 2(\alpha-2) K_{4}^{\left(X_{2}\right)}(\alpha)=\left[2(\alpha-2) u+3 \alpha^{2}-11 \alpha+12\right] u^{3} \frac{\mathrm{d}^{2}}{\mathrm{~d} u^{2}}-\left[12(\alpha-2) u^{3}\right. \\
& \quad-\left(3 \alpha^{3}-36 \alpha^{2}+97 \alpha-84\right) u^{2}-(\alpha-1)\left(7 \alpha^{3}-49 \alpha^{2}+112 \alpha-80\right) u \\
& \left.\quad-2(\alpha-1)\left(\alpha^{4}-11 \alpha^{3}+32 \alpha^{2}-36 \alpha+16\right)\right] \frac{\mathrm{d}}{\mathrm{d} u}+24(\alpha-2) u^{2} \\
& \quad-4\left(3 \alpha^{3}-27 \alpha^{2}+64 \alpha-48\right) u+\frac{4(\alpha-1)}{f(u ; \alpha)}\left\{( \alpha - 1 ) \left[\left(\alpha^{3}-3 \alpha^{2}+8 \alpha-16\right) u\right.\right. \\
& \left.\quad+\alpha\left(\alpha^{3}-3 \alpha^{2}+8 \alpha-16\right)\right] \frac{\mathrm{d}}{\mathrm{d} u}+\alpha\left[\left(\alpha^{3}-3 \alpha^{2}+8 \alpha-16\right) u\right. \\
& \left.\left.\quad+\alpha(\alpha-1)\left(\alpha^{2}-3 \alpha+4\right)\right]\right\}
\end{aligned}
$$

\section{Appendix E: Commutation Relations of $J_{i}$}

Every commutation relation of the quasi-solvable operators $J_{i}(i=1, \ldots, 8)$ is presented in terms of $J_{1}, J_{4}$, and $J_{9}$ :

$$
\begin{aligned}
& {\left[J_{1}, J_{2}\right]=\frac{2}{f^{\prime \prime}} \frac{\mathrm{d}}{\mathrm{d} z} J_{1}, \quad\left[J_{1}, J_{3}\right]=\left(\frac{2 f^{\prime}}{f^{\prime \prime}} \frac{\mathrm{d}}{\mathrm{d} z}+1\right) J_{1}, \quad\left[J_{1}, J_{4}\right]=2 \frac{\mathrm{d}}{\mathrm{d} z} J_{1},} \\
& {\left[J_{1}, J_{5}\right]=2 z \frac{\mathrm{d}}{\mathrm{d} z} J_{1}+\frac{2}{f^{\prime \prime}} \frac{\mathrm{d}}{\mathrm{d} z} J_{4}, \quad\left[J_{1}, J_{6}\right]=2 f \frac{\mathrm{d}}{\mathrm{d} z} J_{1}+\left(\frac{2 f^{\prime}}{f^{\prime \prime}} \frac{\mathrm{d}}{\mathrm{d} z}+1\right) J_{4},} \\
& {\left[J_{1}, J_{7}\right]=z\left(2 z \frac{\mathrm{d}}{\mathrm{d} z}-1\right) J_{1}+\frac{2}{f^{\prime \prime}} \frac{\mathrm{d}}{\mathrm{d} z} J_{9},} \\
& {\left[J_{1}, J_{8}\right]=f\left(2 z \frac{\mathrm{d}}{\mathrm{d} z}-1\right) J_{1}+\left(\frac{2 f^{\prime}}{f^{\prime \prime}} \frac{\mathrm{d}}{\mathrm{d} z}+1\right) J_{9},}
\end{aligned}
$$




$$
\begin{aligned}
& {\left[J_{2}, J_{3}\right]=\left(2 \frac{z f^{\prime}-f}{f^{\prime \prime}} \frac{\mathrm{d}}{\mathrm{d} z}+z\right) J_{1}, \quad\left[J_{2}, J_{4}\right]=\left(2 \frac{z f^{\prime}-f}{f^{\prime \prime}} \frac{\mathrm{d}}{\mathrm{d} z}+1\right) J_{1},} \\
& {\left[J_{2}, J_{5}\right]=z\left(2 \frac{z f^{\prime \prime}-f^{\prime}}{f^{\prime \prime}} \frac{\mathrm{d}}{\mathrm{d} z}+1\right) J_{1}+\frac{2 z}{f^{\prime \prime}} \frac{\mathrm{d}}{\mathrm{d} z} J_{4},} \\
& {\left[J_{2}, J_{6}\right]=f\left(2 \frac{z f^{\prime \prime}-f^{\prime}}{f^{\prime \prime}} \frac{\mathrm{d}}{\mathrm{d} z}+1\right) J_{1}+z\left(\frac{2 f^{\prime}}{f^{\prime \prime}} \frac{\mathrm{d}}{\mathrm{d} z}+1\right) J_{4},} \\
& {\left[J_{2}, J_{7}\right]=2 z \frac{z^{2} f^{\prime \prime}-z f^{\prime}+f}{f^{\prime \prime}} \frac{\mathrm{d}}{\mathrm{d} z} J_{1}+\frac{2 z}{f^{\prime \prime}} \frac{\mathrm{d}}{\mathrm{d} z} J_{9},} \\
& {\left[J_{2}, J_{8}\right]=2 f \frac{z^{2} f^{\prime \prime}-z f^{\prime}+f}{f^{\prime \prime}} \frac{\mathrm{d}}{\mathrm{d} z} J_{1}+z\left(\frac{2 f^{\prime}}{f^{\prime \prime}} \frac{\mathrm{d}}{\mathrm{d} z}+1\right) J_{9},} \\
& {\left[J_{3}, J_{4}\right]=2 \frac{f f^{\prime \prime}-\left(f^{\prime}\right)^{2}}{f^{\prime \prime}} \frac{\mathrm{d}}{\mathrm{d} z} J_{1}, \quad\left[J_{3}, J_{5}\right]=2 z \frac{f f^{\prime \prime}-\left(f^{\prime}\right)^{2}}{f^{\prime \prime}} \frac{\mathrm{d}}{\mathrm{d} z} J_{1}+\frac{2 f}{f^{\prime \prime}} \frac{\mathrm{d}}{\mathrm{d} z} J_{4},} \\
& {\left[J_{3}, J_{6}\right]=2 f \frac{f f^{\prime \prime}-\left(f^{\prime}\right)^{2}}{f^{\prime \prime}} \frac{\mathrm{d}}{\mathrm{d} z} J_{1}+f\left(\frac{2 f^{\prime}}{f^{\prime \prime}} \frac{\mathrm{d}}{\mathrm{d} z}+1\right) J_{4},} \\
& {\left[J_{3}, J_{7}\right]=2 z \frac{z f f^{\prime \prime}-z\left(f^{\prime}\right)^{2}+f f^{\prime}}{f^{\prime \prime}} \frac{\mathrm{d}}{\mathrm{d} z} J_{1}+\frac{2 f}{f^{\prime \prime}} \frac{\mathrm{d}}{\mathrm{d} z} J_{9},} \\
& {\left[J_{3}, J_{8}\right]=2 f \frac{z f f^{\prime \prime}-z\left(f^{\prime}\right)^{2}+f f^{\prime}}{f^{\prime \prime}} \frac{\mathrm{d}}{\mathrm{d} z} J_{1}+f\left(\frac{2 f^{\prime}}{f^{\prime \prime}} \frac{\mathrm{d}}{\mathrm{d} z}+1\right) J_{9},} \\
& {\left[J_{4}, J_{5}\right]=\left(\frac{2 f^{\prime}}{f^{\prime \prime}} \frac{\mathrm{d}}{\mathrm{d} z}-1\right) J_{4}, \quad\left[J_{4}, J_{6}\right]=\frac{2\left(f^{\prime}\right)^{2}}{f^{\prime \prime}} \frac{\mathrm{d}}{\mathrm{d} z} J_{4},} \\
& {\left[J_{4}, J_{7}\right]=2 z f \frac{\mathrm{d}}{\mathrm{d} z} J_{1}-z J_{4}+\left(\frac{2 f^{\prime}}{f^{\prime \prime}} \frac{\mathrm{d}}{\mathrm{d} z}-1\right) J_{9},} \\
& {\left[J_{4}, J_{8}\right]=2 f^{2} \frac{\mathrm{d}}{\mathrm{d} z} J_{1}-f J_{4}+\frac{2\left(f^{\prime}\right)^{2}}{f^{\prime \prime}} \frac{\mathrm{d}}{\mathrm{d} z} J_{9}} \\
& {\left[J_{5}, J_{6}\right]=\left(2 f^{\prime} \frac{z f^{\prime}-f}{f^{\prime \prime}} \frac{\mathrm{d}}{\mathrm{d} z}+f\right) J_{4},} \\
& {\left[J_{5}, J_{7}\right]=2 z^{2} f \frac{\mathrm{d}}{\mathrm{d} z} J_{1}-2 z \frac{z f^{\prime}-f}{f^{\prime \prime}} \frac{\mathrm{d}}{\mathrm{d} z} J_{4}+z\left(\frac{2 f^{\prime}}{f^{\prime \prime}} \frac{\mathrm{d}}{\mathrm{d} z}-1\right) J_{9},} \\
& {\left[J_{5}, J_{8}\right]=2 z f^{2} \frac{\mathrm{d}}{\mathrm{d} z} J_{1}-2 f \frac{z f^{\prime}-f}{f^{\prime \prime}} \frac{\mathrm{d}}{\mathrm{d} z} J_{4}+\frac{2 z\left(f^{\prime}\right)^{2}}{f^{\prime \prime}} \frac{\mathrm{d}}{\mathrm{d} z} J_{9},} \\
& {\left[J_{6}, J_{7}\right]=2 z f^{2} \frac{\mathrm{d}}{\mathrm{d} z} J_{1}-2 z f^{\prime} \frac{z f^{\prime}-f}{f^{\prime \prime}} \frac{\mathrm{d}}{\mathrm{d} z} J_{4}+f\left(\frac{2 f^{\prime}}{f^{\prime \prime}} \frac{\mathrm{d}}{\mathrm{d} z}-1\right) J_{9},} \\
& {\left[J_{6}, J_{8}\right]=2 f^{3} \frac{\mathrm{d}}{\mathrm{d} z} J_{1}-2 f f^{\prime} \frac{z f^{\prime}-f}{f^{\prime \prime}} \frac{\mathrm{d}}{\mathrm{d} z} J_{4}+\frac{2 f\left(f^{\prime}\right)^{2}}{f^{\prime \prime}} \frac{\mathrm{d}}{\mathrm{d} z} J_{9},} \\
& {\left[J_{7}, J_{8}\right]=2 \frac{z^{2}\left(f^{\prime}\right)^{2}-2 z f f^{\prime}+f^{2}}{f^{\prime \prime}} \frac{\mathrm{d}}{\mathrm{d} z} J_{9} .}
\end{aligned}
$$




\section{Appendix F: The Explicit Forms of the Coefficients}

The constant coefficients $C_{i j}(\alpha)(i=1, \ldots, 4 ; j=0, \ldots, 8)$ appeared in (7.18) and (7.23) are explicitly given by

$$
\begin{aligned}
& C_{12}(\alpha)=2(\alpha+3), \quad C_{13}(\alpha)=-2, \quad C_{14}(\alpha)=-(\alpha+2), \\
& C_{15}(\alpha)=1, \quad C_{10}(\alpha)=-2 \text {, } \\
& C_{21}(\alpha)=\frac{2(\alpha+3)(\alpha+2)(\alpha-1)}{\alpha+1}, \quad C_{22}(\alpha)=-\frac{2(\alpha-1)\left(3 \alpha^{2}+12 \alpha+13\right)}{\alpha+1} \\
& C_{23}(\alpha)=\frac{2\left(\alpha^{2}-3 \alpha-2\right)}{\alpha(\alpha+1)}, \quad C_{24}(\alpha)=\frac{(\alpha+2)(\alpha-1)\left(3 \alpha^{2}+6 \alpha+4\right)}{\alpha(\alpha+1)}, \\
& C_{25}(\alpha)=\frac{4(\alpha+2)}{\alpha(\alpha+1)}, \quad C_{26}(\alpha)=-\frac{\alpha}{\alpha+1}, \quad C_{27}(\alpha)=\frac{2(\alpha-1)}{\alpha}, \\
& C_{20}(\alpha)=\frac{2(\alpha-2)(\alpha-1)}{\alpha} \\
& C_{33}(\alpha)=2\left(3 \alpha^{2}+7 \alpha+6\right), \quad C_{35}(\alpha)=-\left(3 \alpha^{2}+5 \alpha+4\right), \quad C_{36}(\alpha)=\alpha, \\
& C_{37}(\alpha)=-2(\alpha-1), \quad C_{30}(\alpha)=4(\alpha+4)(\alpha-1), \\
& C_{42}(\alpha)=-2 \alpha(\alpha+3)^{2}(\alpha-1), \quad C_{43}(\alpha)=-\frac{(\alpha-1)\left(7 \alpha^{3}+31 \alpha^{2}+54 \alpha+36\right)}{\alpha+1}, \\
& C_{44}(\alpha)=\frac{\alpha(\alpha+3)(\alpha+2)^{2}(\alpha-1)}{\alpha+1}, \quad C_{45}(\alpha)=\frac{(\alpha-1)\left(7 \alpha^{3}+31 \alpha^{2}+54 \alpha+48\right)}{2(\alpha+1)}, \\
& C_{46}(\alpha)=-\frac{\left(3 \alpha^{3}-5 \alpha^{2}-14 \alpha-8\right)}{2(\alpha+1)}, \quad C_{47}(\alpha)=\frac{(\alpha-1)\left(3 \alpha^{2}+\alpha-12\right)}{\alpha+1}, \\
& C_{48}(\alpha)=\frac{2(\alpha-1)}{\alpha+1}, \quad C_{40}(\alpha)=-\frac{4(\alpha-1)\left(\alpha^{3}+7 \alpha^{2}-10\right)}{\alpha+1} .
\end{aligned}
$$

The other coefficients which are not appeared above all vanish.

[1] H. Aoyama, M. Sato, and T. Tanaka, Nucl. Phys. B 619 (2001) 105. arXiv:quant-ph/0106037.

[2] H. Aoyama, N. Nakayama, M. Sato, and T. Tanaka, Phys. Lett. B 519 (2001) 260. arXiv:hepth/0107048.

[3] T. Tanaka, Nucl. Phys. B 662 (2003) 413. arXiv:hep-th/0212276.

[4] A. González-López and T. Tanaka, J. Phys. A: Math. Gen. 38 (2005) 5133. arXiv:hepth/0405079.

[5] A. González-López and T. Tanaka, Phys. Lett. B 586 (2004) 117. arXiv:hep-th/0307094.

[6] D. Gómez-Ullate, N. Kamran, and R. Milson, Inverse Problems 23 (2007) 1915. arXiv:nlin/0610065.

[7] D. Gómez-Ullate, N. Kamran, and R. Milson, J. Approx. Theory 162 (2010) 987. arXiv:0805.3376 [math-ph]. 
[8] T. Tanaka, J. Math. Phys. 51 (2010) 032101. arXiv:0910.0328 [math-ph].

[9] T. Tanaka, In Morris B. Levy, ed., Mathematical Physics Research Developments (Nova Science Publishers, Inc., New York, 2009), chapter 18. pp. 621-679.

[10] T. Tanaka, In D. E. Hathaway and E. M. Randolph, eds., Focus on Quantum Mechanics (Nova Science Publishers, Inc., New York, 2011), chapter 12. pp. 345-403.

[11] T. Tanaka, J. Phys. A: Math. Theor. 44 (2011) 465301. arXiv:1107.1035 [math-ph].

[12] A. González-López and T. Tanaka, J. Phys. A: Math. Gen. 39 (2006) 3715. arXiv:quant$\mathrm{ph} / 0602177$.

[13] B. Bagchi and T. Tanaka, Ann. Phys. 324 (2009) 2438. arXiv:0905.4330 [hep-th].

[14] B. Roy and T. Tanaka. Two-step shape invariance in the framework of $\mathcal{N}$-fold supersymmetry. arXiv:1203.4012 [math-ph].

[15] N. Kamran, R. Milson, and P. J. Olver, Adv. Math. 156 (2000) 286.

[16] A. González-López, N. Kamran, and P. J. Olver, Commun. Math. Phys. 153 (1993) 117.

[17] G. Post and A. Turbiner, Russ. J. Math. Phys. 3 (1995) 113. arXiv:funct-an/9307001.

[18] H. Aoyama, M. Sato, and T. Tanaka, Phys. Lett. B 503 (2001) 423. arXiv:quant-ph/0012065.

[19] A. V. Turbiner, Commun. Math. Phys. 118 (1988) 467.

[20] A. González-López, N. Kamran, and P. J. Olver, Contemp. Math. 160 (1994) 113.

[21] A. V. Turbiner, Contemp. Math. 160 (1994) 263.

[22] D. Gómez-Ullate, N. Kamran, and R. Milson, J. Phys. A: Math. Gen. 38 (2005) 2005. arXiv:nlin/0401030.

[23] L. É. Gendenshteîn, JETP Lett. 38 (1983) 356.

[24] D. T. Barclay, R. Dutt, A. Gangopadhyaya, A. Khare, A. Pagnamenta, and U. Sukhatme, Phys. Rev. A 48 (1993) 2786. arXiv:hep-ph/9304313.

[25] B. Bagchi and T. Tanaka, Ann. Phys. 325 (2010) 1679. arXiv:1002.1766 [hep-th]. 